\title{
Identification of sources and processes in a low-level radioactive waste site adjacent to landfills: groundwater hydrogeochemistry and isotopes
}

D. I. CENDÓN $N^{1,2,3^{*}}$, C. E. HUGHES ${ }^{1}$, J. J. HARRISON ${ }^{1}$, S. I. HANKIN ${ }^{1}$, M. P.

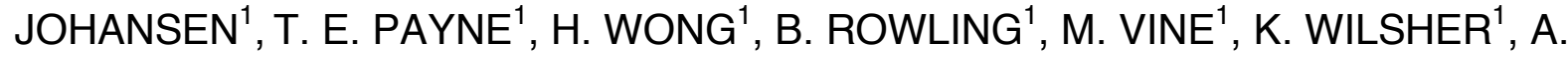
GUINEA $^{4,3}$ AND S. THIRUVOTH ${ }^{1}$

${ }^{1}$ Australian Nuclear Science and Technology Organisation, Locked Bag 2001, Kirrawee, NSW 2232, Australia

${ }^{2}$ School of Biological Earth and Environmental Sciences, The University of New South Wales, Sydney, NSW 2052, Australia

${ }^{3}$ Connected Waters Initiative, School of Biological, Earth and Environmental Sciences, The University of New South Wales, Sydney, NSW 2052, Australia ${ }^{4}$ School of Applied and Biomedical Sciences, Federation University, Mt Helen, VIC, 3350, Australia

(*) Corresponding author contact: dce@ansto.gov.au; Tel: +61 297173937

Australian Journal of Earth Sciences (2015) 62, 123-141

http://dx.doi.org/10.1080/08120099.2015.975155

Copies of Supplementary Papers may be obtained from the Geological Society of Australia's website (www.gsa.org.au), the Australian Journal of Earth Scienecs website (www.ajes.com.au) or from the National Library of Australia's Pandora archive (http://nla.gov.au/nla.arc-25194). 


\section{SUPPLEMENTARY PAPERS}

Supplementary Paper S1 Electrical Resistivity Tomography (ERT) profiles.

Supplementary Paper S2 Details of well construction.

Supplementary Paper S3 Groundwater major physico-chemical parameters, ion concentrations and charge balance error (CBE\%). All cations analysed by ICP-AES except for $\mathrm{Mg}^{2+}$ in the first 3 samplings analysed by ICP-MS.

Supplementary Paper S4 Groundwater and surface water information with well location, screened interval and abbreviated water type.

Supplementary Paper S5 Selected groundwater minor and trace element concentrations. AI, Mn and Fe analysed by ICP-AES, the rest of elements by ICP_MS. BDL (bellow detection limit); no data represent not analysed elements.

Supplementary Paper S6 Groundwater dissolved organic carbon (DOC), stable isotopes $\left(\delta^{13} \mathrm{C}_{\text {DOC }}, \delta^{13} \mathrm{C}_{\text {DIC }}\right.$, water $\delta^{18} \mathrm{O}$ and $\delta^{2} \mathrm{H}$, sulfate $\delta^{34} S$ and $\left.\delta^{18} \mathrm{O}\right)$, calculated deuterium excess (d-excess), ${ }^{87} \mathrm{Sr} /{ }^{86} \mathrm{Sr}$ and tritium $\left({ }^{3} \mathrm{H}\right)$. Note ${ }^{3} \mathrm{H}$ is reported in $\mathrm{Bq} \mathrm{L}^{-1}\left(1 \mathrm{~Bq} \mathrm{~L}^{-1}=8.47 \mathrm{TU}\right)$.

Supplementary Paper S7 Water stable isotopes for leachate samples from Harrington's Quarry. The $d$ is the calculated deuterium excess for the corresponding samples.

Supplementary Paper S8 Calculated saturation indexes for common mineral phases in LFLS groundwater. $\mathrm{Cal}=$ Calcite, $\mathrm{Dol}=$ Dolomite, $\mathrm{Sid}=$ Siderite, Gyp = Gypsum, Hem = Hematite, Goe = Goethite, Qtz = Quartz, Bar = Barite, Cha = Chalcedony, $\mathrm{Hal}=$ Halite, $\mathrm{Illi}=\mathrm{Illite}, \mathrm{KaO}=$ Kaolinite . 
Supplementary Paper S1 Electrical Resistivity Tomography (ERT) profiles. An Electrical Resistivity Tomography (ERT) campaign was carried out using a SAS 4000 Terrameter with ABEM Lund system (Dahlin 1996). The Wenner-Schlumberger electrode array was selected due to the lateral continuity of the layers (Szalai et al. 2009). The apparent resistivity data was inverted with the software RES2DINV (Loke et al. 1996).

In the resistivity sections, Hawkesbury Sandstone is indicated at depth displaying an electrical resistivity above $160 \mathrm{ohm} . \mathrm{m}$, with the central part of profile 1 (Figure A1), having a lower resistivity. This corresponds with materials generally grading conformably upwards from the Hawkesbury sandstone and overlaid by the shale lens. Above the Hawkesbury Sandstone, the shale displays lower electrical resistivity (20-30 ohm.m) with a thickness of approximately 14 metres along the studied sections. The deepening of this layer towards North is seen in profile 1 , while profiles 2 and 3 show a similar thickness (Figure A1). Drilling during BH well construction was used to map the shale layer and its thickness (Mumme 1974) with a good agreement with the ERT profiles. The shale mapping showed a thinning of the shale layer to the NW of the site. The soil layer is more resistive than the shale (120-150 ohm.m) and more heterogeneous; this is better shown in profiles 2 and 3 (Figure A1b, c). These heterogeneities are typical in soils due to their unconsolidated nature, and enhanced by the presence of trenches. The soil layer is very thin at the south of the studied area and thickens towards the north, following the dipping of the shale lens.
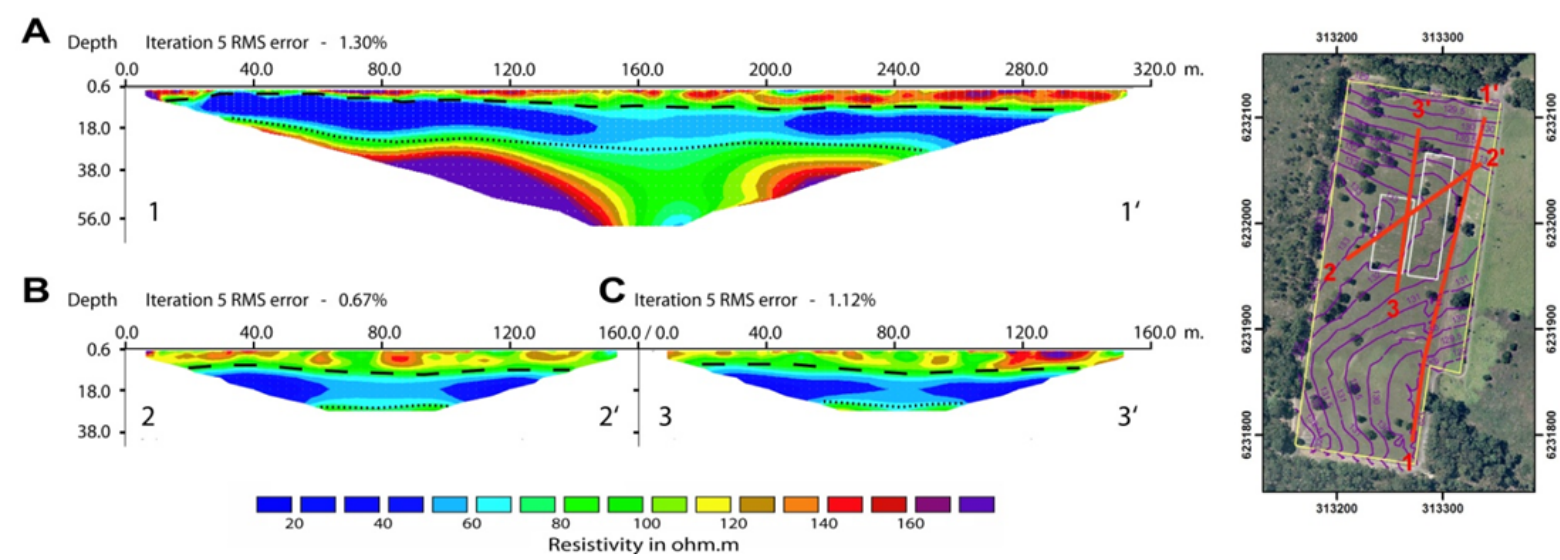

Figure SP1 (A, B, C) Electrical resistivity profiles with location of transects within the LFLS site. Profiles are oriented following the numeric legend in the profiles. The electrode spacing was $5 \mathrm{~m}$ with a total of 64 electrodes for profile 1 and 32 electrodes for profiles 2 and 3. 


\section{Supplementary Paper S2 Details of well construction.}

Detailed information on well construction is given in Hughes et al. (2011) and multiple reports cited therein. In summary, several generations of observation bores are present on the LFLS (Figure 1c). The earliest bores, numbered "BH" or "OS", were established in the 1960s prior to, or during, waste disposal operations (AAEC 1966). These are unscreened open bores partially lined with asbestos cement with the exception of BHF, which is PVC-lined and suspected to have been installed in 1984. The "D" series, established in the mid 1970s, are PVC-lined boreholes mostly $3 \mathrm{~m}$ depth (up to $6 \mathrm{~m}$ ), screened from the surface and mostly located around the trench area (Isaacs \& Mears 1977). No information is available on the type of gravel pack used in the BH, OS and D series wells. The "MB" series established in 1988 covers the fenced perimeter and has a key borehole in the centre between both trench areas (MB16). The MB wells are variable in depth (4-7 $\mathrm{m}$ ) with most cutting through the weathered shale and probably stopping with core refusal at a harder level (sideriterich mudstone or parent shale). The MB series was fitted with basalt gravel-packs. $\mathrm{P} 1 \mathrm{~s}$ is the only borehole screened entirely within the shale layer. $\mathrm{CW}$ is screened in a fine sandstone layer intercalated within the shale, with potentially more shale below the screen. The P1d and P2d are the only boreholes that are screened within the underlying Hawkesbury sandstone down to 22 and $36 \mathrm{~m}$, respectively. A number of wells were developed during 2009 ( $\mathrm{CH}$ series) with screens in the zone $2.5-4.5 \mathrm{~m}$ deep, just below an indurated mudstone layer. The "P", " $\mathrm{CW}$ " and " $\mathrm{CH}$ " series were developed according to Australian borehole construction guidelines (LWBC 2003).

\section{ADDITIONAL REFERENCES}

DAHLIN T. 1996. 2D resistivity surveying for environmental and engineering applications. First Break 14, 275-283.

LOKE M. H. \& BARKER R. D. 1996. Rapid least-squares inversion of apparent resistivity pseudosections by a quasi-Newton method. Geophysical Prospecting 44,131152.

LWBC 2003. Minimum construction requirements for water bores in Australia. 2nd edition. Land and Water Biodiversity Committee. ISBN 1-9209-2009-0.

Szalai S., Novák A. \& SzARKA L. 2009. Depth of investigation and vertical resolution of surface geoelectric arrays. Journal of Environmental and Engineering Geophysics 14, 15-23. 
Cendón et al. 2015 Supplementary Papers

Australian Journal of Earth Sciences 62, 123-141. http://dx.doi.org/10.1080/08120099.2015.975155

Supplementary Paper S3: Groundwater major physico-chemical parameters, ion concentrations and charge balance error (CBE\%). All ratinne analvesed hy IC.P.AFS oxrent for $\mathrm{Mn}^{2+}$ in the firct 3 camnlince analvesed hy IC.P_M.S

\begin{tabular}{|c|c|c|c|c|c|c|c|c|c|c|c|c|c|c|c|c|c|}
\hline & $\begin{array}{c}\text { Temp } \\
{ }^{\circ} \mathrm{C} \\
\end{array}$ & pH & $\begin{array}{c}\text { TDS } \\
\mathrm{mg} \mathrm{L}^{-1}\end{array}$ & $\begin{array}{l}\text { Eh } \\
\mathrm{mV}\end{array}$ & $\begin{array}{c}\mathrm{DO} \\
\mathrm{mg} \mathrm{L}^{-1}\end{array}$ & $\begin{array}{c}\mathrm{Na}^{+} \\
\mathrm{mg} \mathrm{L}^{-1}\end{array}$ & $\begin{array}{c}\mathrm{K}^{+} \\
\mathrm{mg} \mathrm{L}^{-1}\end{array}$ & $\begin{array}{c}\mathrm{Ca}^{2+} \\
\mathrm{mg} \mathrm{L}^{-1}\end{array}$ & $\begin{array}{c}\mathrm{Mg}^{2+} \\
\mathrm{mg} \mathrm{L}^{-1}\end{array}$ & $\begin{array}{c}F^{-} \\
\mathrm{mg} \mathrm{L}^{-1}\end{array}$ & $\begin{array}{c}\mathrm{Cl}^{-} \\
\mathrm{mg} \mathrm{L}^{-1}\end{array}$ & $\begin{array}{c}\mathrm{Br}^{-} \\
\mathrm{mg} \mathrm{L}^{-1}\end{array}$ & $\begin{array}{l}\mathrm{HCO}_{3}^{-} \\
\mathrm{mg} \mathrm{L}^{-1}\end{array}$ & $\begin{array}{c}\mathrm{NO}_{3}^{-} \\
\mathrm{mg} \mathrm{L}^{-1}\end{array}$ & $\begin{array}{c}\mathrm{SO}_{4}{ }^{2-} \\
\mathrm{mg} \mathrm{L}^{-1}\end{array}$ & $\begin{array}{c}\mathbf{S i} \\
\mathrm{mg} \mathrm{L}^{-1}\end{array}$ & CBE \% \\
\hline 2007 & & & & & & & & & & & & & & & & & \\
\hline DCE-07-02-BH10 & 15.6 & 5.27 & 155 & 424 & 0.24 & 52.1 & 0.98 & 0.66 & 1.0 & 0.05 & 66 & 0.65 & 14.0 & 0.1 & 19 & 7.2 & -1.7 \\
\hline DCE-07-01-BHF & 16.9 & 5.18 & 346 & 253 & 0.30 & 96.6 & 3.78 & 2.90 & 12.0 & 0.06 & 201 & 0.67 & 8.5 & $<0.1$ & 20 & 4.2 & -6.8 \\
\hline DCE-07-19-CW & 18.9 & 5.67 & 1518 & 289 & 1.80 & 488 & 7.98 & 8.65 & 23.6 & 1.00 & 580 & 3.1 & 106.1 & 0.1 & 300 & 23.2 & -1.3 \\
\hline DCE-07-05-MB11 & 18.7 & 5.22 & 150 & 393 & 2.20 & 41.5 & 0.67 & 3.22 & 6.8 & 0.05 & 62 & 0.38 & 6.7 & 0.3 & 28 & 7.5 & 2.0 \\
\hline DCE-07-06-MB12 & 16.5 & 5.14 & 172 & 383 & 1.65 & 45.5 & 1.20 & 4.67 & 5.0 & 0.04 & 26 & 0.24 & 16.5 & $<0.1$ & 73 & 18.8 & 2.6 \\
\hline DCE-07-07-MB13 & 15.5 & 5.16 & 103 & 387 & 0.37 & 28.3 & 1.37 & 2.66 & 2.9 & 0.04 & 33 & 0.23 & 11.6 & 0.3 & 23 & 6.4 & 1.4 \\
\hline DCE-07-08-MB14 & 17.3 & 5.71 & 444 & 281 & 1.18 & 117 & 4.44 & 9.81 & 22.0 & 0.07 & 200 & 0.61 & 23.5 & 0.3 & 66 & 9.1 & 0.6 \\
\hline DCE-07-09-MB15 & 18.5 & 5.72 & 808 & 356 & 0.71 & 248 & 3.32 & 8.46 & 8.5 & 0.49 & 155 & 1.2 & 12.3 & 0.4 & 370 & 23.9 & -1.2 \\
\hline DCE-07-10-MB16 & 16.5 & 5.38 & 148 & 294 & 0.32 & 43.0 & 0.91 & 2.42 & 2.9 & 0.07 & 29 & 1.1 & 35.7 & 0.1 & 33 & 13.9 & 3.4 \\
\hline DCE-07-11-MB17 & 15.5 & 5.37 & 139 & 410 & 0.31 & 39.3 & 0.46 & 1.87 & 2.0 & 0.04 & 40 & 0.6 & 32.9 & $<0.1$ & 22 & 8.1 & -3.6 \\
\hline DCE-07-12-MB18 & 18.1 & 6.18 & 415 & 343 & 1.80 & 87.1 & 1.92 & 8.41 & 11.8 & 0.33 & 180 & 1.2 & 14.6 & $<0.1$ & 110 & 10.9 & -18.6 \\
\hline DCE-07-13-MB19 & 16.6 & 5.81 & 2210 & 188 & 0.43 & 580 & 14.7 & 97.3 & 74.7 & 0.08 & 1080 & 3.5 & 150.1 & 0.1 & 210 & 13.9 & -1.1 \\
\hline DCE-07-14-MB20 & 13.7 & 5.65 & 221 & 242 & 0.47 & 39.8 & 9.10 & 18.80 & 8.0 & 0.05 & 68 & 0.33 & 50.8 & 0.1 & 26 & 4.5 & 3.7 \\
\hline DCE-07-15-MB21 & 14.4 & 6.04 & 203 & 275 & 0.53 & 52.8 & 3.99 & 7.61 & 4.9 & 0.03 & 63 & 0.33 & 38.3 & 0.4 & 32 & 4.7 & 1.6 \\
\hline DCE-07-03-OS2 & 13.0 & 5.83 & 131 & 288 & 3.90 & 29.6 & 2.30 & 10.60 & 2.9 & $<0.01$ & 39 & 0.35 & 32.6 & 0.7 & 14 & 5.5 & 4.5 \\
\hline DCE-07-04-OS3 & 16.0 & 5.37 & 110 & 357 & 0.26 & 33.3 & 0.59 & 2.40 & 1.4 & 0.01 & 32 & 0.39 & 21.9 & 0.2 & 18 & 7.1 & 1.8 \\
\hline DCE-07-17-P1D & 18.7 & 5.34 & 6309 & 235 & 0.65 & 1953 & 8.30 & 41.28 & 338.2 & $<0.01$ & 3500 & 10.0 & 68.6 & 0.1 & 390 & 19.9 & 3.0 \\
\hline DCE-07-16.P1S & 17.9 & 4.40 & 2056 & 359 & 0.56 & 800 & 6.56 & 7.99 & 88.2 & 0.18 & 700 & 2.10 & - & 0.5 & 450 & 36.6 & 19.1 \\
\hline $\begin{array}{c}\text { DCE-07-18-P2D } \\
\text { Mar-08 }\end{array}$ & 18.5 & 4.91 & 3196 & 340 & 0.74 & 1060 & 17.8 & 29.20 & 129.3 & 0.60 & 1500 & 5.20 & 44.3 & $<0.1$ & 410 & 21.6 & 6.9 \\
\hline DCE-08-14-BH10 & 20.0 & 5.00 & 288 & 295 & 0.71 & 90.1 & 1.83 & 2.50 & 3.1 & 0.06 & 129 & 1.8 & 11.6 & $<0.01$ & 48 & 17.4 & -5.3 \\
\hline DCE-08-33-BHA & 18.0 & 7.58 & 1849 & 24 & 0.86 & 420 & 11.2 & 35.70 & 52.3 & 0.30 & 766 & 2.1 & 468.2 & & 93 & 2.7 & -11.4 \\
\hline DCE-08-13-BHF & 18.0 & 5.40 & 560 & 250 & 0.37 & 163 & 6.39 & 3.80 & 10.8 & 0.10 & 241 & 1.4 & 34 & $<0.01$ & 96 & 12.0 & -5.2 \\
\hline DCE-08-31-CW & 19.1 & 5.49 & 1734 & 329 & 2.44 & 489 & 10.5 & 10.70 & 29.1 & 1.10 & 779 & 3.7 & 108.3 & $<0.1$ & 302 & 23.6 & -10.3 \\
\hline DCE-08-17-MB11 & 21.4 & 5.18 & 213 & 354 & 0.90 & 58.0 & 1.26 & 3.99 & 8.5 & 0.04 & 116 & 0.42 & 6.5 & 0.08 & 18 & 9.7 & -4.0 \\
\hline DCE-08-18-MB12 & 20.0 & 5.42 & 175 & 286 & 0.96 & 41.4 & 1.55 & 6.82 & 5.6 & 0.04 & 42 & 0.27 & 11.9 & 0.04 & 66 & 15.9 & -2.0 \\
\hline DCE-08-19-MB13 & 20.6 & 4.93 & 163 & 349 & 0.62 & 41.9 & 1.95 & 5.13 & 7.0 & 0.04 & 57 & 0.40 & 10.5 & 0.25 & 39 & 14.5 & 2.6 \\
\hline DCE-08-20-MB14 & 19.9 & 5.03 & 542 & 353 & 1.90 & 119 & 5.07 & 12.10 & 28.5 & 0.07 & 248 & 0.76 & 9.1 & 0.08 & 111 & 15.0 & -4.9 \\
\hline DCE-08-21-MB15 & 21.4 & 5.26 & 232 & 335 & 1.58 & 58.5 & 1.21 & 7.06 & 8.3 & 0.06 & 53 & 0.54 & 22.6 & 0.15 & 81 & 22.3 & 0.8 \\
\hline DCE-08-22-MB16 & 20.3 & 5.20 & 134 & 297 & 0.37 & 37.3 & 1.02 & 3.37 & 3.3 & 0.06 & 31 & 1.0 & 23.5 & 0.14 & 33 & 13.3 & 3.8 \\
\hline DCE-08-23-MB17 & 21.5 & 5.03 & 150 & 326 & 0.51 & 45.3 & 0.62 & 2.31 & 2.2 & 0.05 & 42 & 0.99 & 17.6 & $<0.01$ & 39 & 17.7 & 0.1 \\
\hline DCE-08-24-MB18 & 20.6 & 6.01 & 854 & 305 & 2.42 & 186 & 5.13 & 39.80 & 21.0 & 0.40 & 268 & 2.0 & 119.0 & $<0.1$ & 213 & 15.6 & -7.9 \\
\hline DCE-08-25-MB19 & 17.5 & 5.78 & 1712 & 167 & 1.11 & 368 & 13.4 & 68.2 & 60.8 & 0.17 & 895 & 3.0 & 144.0 & $<0.1$ & 156 & 12.9 & -10.9 \\
\hline
\end{tabular}


Cendón et al. 2015 Supplementary Papers

Australian Journal of Earth Sciences 62, 123-141. http://dx.doi.org/10.1080/08120099.2015.975155

\begin{tabular}{|c|c|c|c|c|c|c|c|c|c|c|c|c|c|c|c|c|c|}
\hline & Temp & pH & TDS & Eh & DO & $\mathrm{Na}^{+}$ & $\mathrm{K}^{+}$ & $\mathrm{Ca}^{2+}$ & $\mathbf{M g}^{2+}$ & $\mathbf{F}^{-}$ & $\mathrm{Cl}^{-}$ & $\mathrm{Br}^{-}$ & $\mathrm{HCO}_{3}^{-}$ & $\mathrm{NO}_{3}{ }^{-}$ & $\mathrm{SO}_{4}{ }^{2-}$ & Si & CBE \% \\
\hline & ${ }^{\circ} \mathrm{C}$ & & $\mathrm{mg} \mathrm{L}^{-1}$ & $\mathbf{m V}$ & $\mathrm{mg} \mathrm{L}^{-1}$ & $\mathrm{mg} \mathrm{L}^{-1}$ & $\mathrm{mg} \mathrm{L}^{-1}$ & $\mathrm{mg} \mathrm{L}^{-1}$ & $\mathrm{mg} \mathrm{L}^{-1}$ & $\mathrm{mg} \mathrm{L}^{-1}$ & $\mathrm{mg} \mathrm{L}^{-1}$ & $\mathrm{mg} \mathrm{L}^{-1}$ & $\mathrm{mg} \mathrm{L}^{-1}$ & $\mathrm{mg} \mathrm{L}^{-1}$ & $\mathrm{mg} \mathrm{L}^{-1}$ & $\mathrm{mg} \mathrm{L}^{-1}$ & \\
\hline DCE-08-26-MB20 & 17.5 & 5.82 & 364 & 235 & 0.51 & 49.9 & 14.1 & 31.40 & 13.2 & 0.06 & 70 & 0.53 & 121.4 & 0.1 & 60 & 10.7 & 0.7 \\
\hline DCE-08-27-MB21 & 18.6 & 5.82 & 1009 & 284 & 0.44 & 187 & 10.3 & 67.6 & 35.0 & $<0.1$ & 305 & 1.5 & 102.8 & $<0.1$ & 299 & 11.0 & -6.1 \\
\hline DCE-08-32-MB22 & 21.5 & 6.02 & 387 & 294 & 3.17 & 77.8 & 2.66 & 10.30 & 27.3 & 0.03 & 140 & 0.38 & 97.7 & $<0.01$ & 15 & 7.0 & 7.0 \\
\hline DCE-08-15-OS2 & 1.5 & 6.02 & 227 & 294 & 3.17 & 33.2 & 3.13 & 28.60 & 4.1 & 0.04 & 38 & 0.46 & 98.0 & 0.31 & 21 & 11.7 & 2.5 \\
\hline DCE-08-16-OS3 & 20.5 & 5.45 & 303 & 160 & 0.26 & 57.6 & 1.67 & 12.50 & 5.1 & 0.02 & 32 & 1.1 & 128.8 & $<0.01$ & 51 & 21.4 & -1.0 \\
\hline DCE-08-28-P1D & 18.6 & 5.15 & 6806 & 284 & 0.50 & 1810 & 8.68 & 41.60 & 376.7 & 0.40 & 4030 & 11.0 & 28.7 & $<0.1$ & 419 & 20.6 & -3.4 \\
\hline DCE-O\& & 20.1 & 4.00 & 3392 & 403 & 0.58 & 898 & 8.05 & 8.73 & 120.6 & 0.50 & 1860 & 4.8 & 8.9 & $<0.1$ & 470 & 41.1 & -10.9 \\
\hline $\begin{array}{l}\text { DCE-08-30-P2D } \\
\text { Sep-Nov } 2008\end{array}$ & 19.3 & 5.62 & 1983 & 252 & 1.26 & 548 & 12.9 & 14.50 & 52.5 & 0.60 & 1000 & 2.9 & 67.3 & $<0.1$ & 269 & 18.1 & -8.1 \\
\hline DCE-08 & 15.0 & 5.31 & 482 & 170 & 9.94 & 136 & 2.16 & 2.16 & 5.6 & 0.08 & 150 & 2.9 & 40.4 & $<0.05$ & 140 & 12.3 & -8.5 \\
\hline DCE-O\& & 18.0 & 7.96 & 3536 & -75 & 0.55 & 972 & 15.7 & 53.8 & 90.9 & 0.23 & 2000 & 5.8 & 286.2 & $<0.05$ & 110 & 3.7 & -10.1 \\
\hline$-176-B H B$ & 17.5 & 7.15 & 5297 & -61 & 0.29 & 1310 & 21 & 123 & 256.5 & 0.36 & 3300 & 22 & 85.4 & $<0.05$ & 130 & 1.6 & -6.7 \\
\hline DCE & 17.6 & 4.91 & 572 & 329 & 6.00 & 5 & 4.76 & 3.59 & 14.0 & 0.12 & 300 & 1.6 & 13.4 & $<0.05$ & 68 & 12.0 & -7.5 \\
\hline DCE-08 & 19.0 & 5.58 & 1852 & 268 & 3.36 & 556 & 9.86 & 10.60 & 31.5 & 1.20 & 760 & 3.9 & 138.7 & $<0.05$ & 340 & 21.0 & -5.7 \\
\hline -160-MB11 & 8.9 & 5.24 & 121 & 279 & 2.58 & 31.7 & 0.57 & 2.23 & 3.9 & 0.05 & 25 & 0.41 & 12.4 & $<0.05$ & 45 & 9.8 & -0.6 \\
\hline DCE & 6.3 & 5.02 & 201 & 267 & 4.16 & 52.2 & 1.12 & 5.28 & 6.6 & 0.04 & 39 & 0.26 & 11.0 & $<0.05$ & 86 & 16.3 & 0.6 \\
\hline DCE-C & 15.7 & 5.20 & 141 & 349 & 3.11 & 39.8 & 1.44 & 3.91 & 6.1 & 0.05 & 40 & 0.29 & 13.4 & $<0.05$ & 36 & 10.8 & 8.2 \\
\hline -163-MB14 & 7.0 & 5.61 & 543 & 238 & 3.64 & 139 & 3.45 & 8.69 & 26.3 & 0.09 & 240 & 0.64 & 24.5 & $<0.05$ & 98 & 21.3 & -2.3 \\
\hline DCE & & 5.26 & 240 & 313 & 2.14 & 63.8 & 1.19 & 7.40 & 8.8 & 0.07 & 61 & 0.44 & 22.1 & $<0.05$ & 75 & 22.9 & 3.5 \\
\hline MB16 & 17.2 & 5.17 & 131 & 249 & 1.13 & 34.5 & 0.71 & 2.34 & 2.8 & 0.06 & 27 & 0.84 & 29.2 & 0.14 & 33 & 9.1 & -1.3 \\
\hline -166-MB17 & 17.5 & 5.17 & 193 & 302 & 2.99 & 53.7 & 0.66 & 4.16 & 3.1 & 0.04 & 36 & 1.3 & 16.0 & $<0.05$ & 78 & 24.1 & -1.6 \\
\hline & & 6.23 & 236 & 237 & 2.47 & 59.1 & & 7.06 & 4.3 & 0.19 & 59 & 0.31 & & & 30 & 10.6 & -3.2 \\
\hline -168-MB19 & 16.5 & 5.77 & 1656 & 270 & 1.11 & 416 & 12.8 & 62.90 & 46.4 & 0.17 & 810 & 2.8 & 101.8 & 0.31 & 200 & 13.9 & -6.1 \\
\hline DCE-08-169-MB20 & 15.6 & 5.90 & 185 & 162 & 3.07 & 31.9 & 7.79 & 13.80 & 4.8 & 0.07 & 35 & 0.31 & 62.9 & 0.16 & 28 & 5.8 & 1.3 \\
\hline & & 5.16 & 145 & 238 & & 36.6 & & & 3.9 & $<0.05$ & 50 & & 24.2 & 0.20 & 21 & 3.0 & 0.0 \\
\hline DCE-C & 17.6 & 6.34 & 148 & 231 & 7.38 & 29.7 & 2.25 & 12.20 & 2.9 & $<0.05$ & 35 & 0.43 & 41.6 & 1.00 & 23 & 4.7 & 1.0 \\
\hline DCE-08-159-OS3 & 17.3 & 4.97 & 106 & 324 & 4.53 & 29.7 & 0.45 & 1.63 & 1.4 & $<0.05$ & 29 & 0.49 & 14.6 & $<0.05$ & 23 & 5.5 & -0.4 \\
\hline DCE-08-171-P1D & 19 & 5.39 & 7081 & 193 & 0.74 & 1880 & 7.95 & 39.20 & 377.2 & 0.28 & 4200 & 9.8 & 59.0 & $<0.05$ & 480 & 19.3 & -5.6 \\
\hline & & 3.99 & 4063 & 417 & 1.82 & 1280 & & & 109.9 & 0.44 & 2100 & 5.9 & 9.2 & $<0.05$ & 530 & 33.9 & -3.4 \\
\hline DCE-08-173-P2D & 20.3 & 5.81 & 2639 & 213 & 1.74 & 756 & 14.1 & 18.90 & 67.9 & 0.61 & 1300 & 4.0 & 124.8 & $<0.05$ & 350 & 16.0 & -7.3 \\
\hline DCE-08-177-D14 & 19.2 & 4.43 & 180 & 332 & 2.96 & 52.0 & 2.15 & 0.53 & 0.0 & $<0.05$ & 45 & 0.70 & 5.9 & $<0.05$ & 73 & 24.2 & -9.5 \\
\hline IDCE-08-180-JTC & 14.7 & 4.85 & 458 & 287 & 7.18 & 119 & 6.53 & 3.62 & 15.8 & 0.07 & 210 & 0.89 & 3.5 & $<0.05$ & 98 & 3.4 & -7.9 \\
\hline
\end{tabular}


Cendón et al. 2015 Supplementary Papers

Australian Journal of Earth Sciences 62, 123-141. http://dx.doi.org/10.1080/08120099.2015.975155

\begin{tabular}{|c|c|c|c|c|c|c|c|c|c|c|c|c|c|c|c|c|c|}
\hline & Temp & $\mathrm{pH}$ & TDS & Eh & DO & $\mathrm{Na}^{+}$ & $\mathrm{K}^{+}$ & $\mathrm{Ca}^{2+}$ & $\mathrm{Mg}^{2+}$ & $\mathbf{F}^{-}$ & $\mathrm{Cl}^{-}$ & $\mathrm{Br}^{-}$ & $\mathrm{HCO}_{3}^{-}$ & $\mathrm{NO}_{3}{ }^{-}$ & $\mathrm{SO}_{4}{ }^{2-}$ & $\mathbf{S i}$ & CBE \% \\
\hline & ${ }^{\circ} \mathrm{C}$ & & $\mathrm{mg} \mathrm{L}^{-1}$ & $\mathrm{mV}$ & $\mathrm{mg} \mathrm{L}^{-1}$ & $\mathrm{mg} \mathrm{L}^{-1}$ & $\mathrm{mg} \mathrm{L}^{-1}$ & $\mathrm{mg} \mathrm{L}^{-1}$ & $\mathrm{mg} \mathrm{L}^{-1}$ & $\mathrm{mg} \mathrm{L}^{-1}$ & $\mathrm{mg} \mathrm{L}^{-1}$ & $\mathrm{mg} \mathrm{L}^{-1}$ & $\mathrm{mg} \mathrm{L}^{-1}$ & $\mathrm{mg} \mathrm{L}^{-1}$ & $\mathrm{mg} \mathrm{L}^{-1}$ & $\mathrm{mg} \mathrm{L}^{-1}$ & \\
\hline \multicolumn{18}{|l|}{2009} \\
\hline DCE-09-77-BH10 & 18.6 & 5.41 & 323 & 231 & 3.56 & 78.2 & 1.33 & 0.87 & 1.82 & 0.05 & 91 & 1.3 & 112.1 & $<0.1$ & 36 & 15.6 & 1.8 \\
\hline DCE-09-76-BHF & 20.5 & 5.28 & 979 & 291 & 0.64 & 278 & 7.16 & 6.13 & 20.8 & 0.18 & 460 & 3.0 & 21.0 & 0.1 & 180 & 13.0 & -8.6 \\
\hline DCE-09-94-CW & 26.5 & 5.54 & 1656 & 323 & 2.05 & 513 & 9.48 & 10.50 & 25.4 & 1.50 & 640 & 3.5 & 122.7 & $<0.1$ & 330 & 23.8 & -3.5 \\
\hline DCE-09-80-MB11 & 20.0 & 5.23 & 271 & 360 & 2.35 & 81.1 & 1.00 & 5.88 & 9.02 & 0.06 & 140 & 0.49 & 13.5 & $<0.1$ & 20 & 10.9 & 0.0 \\
\hline DCE & 23 & 5.09 & 271 & 378 & 1.91 & 72.9 & 1.17 & 6.95 & 6.7 & 0.06 & 45 & 0.32 & 7.8 & $<0.1$ & 130 & 28.9 & 0.0 \\
\hline DCE-09-82-MB13 & 24.5 & 5.22 & 104 & 388 & 1.79 & 31.1 & 1.03 & 2.13 & 2.18 & 0.05 & 30 & 0.23 & 6.2 & $<0.1$ & 31 & 13.0 & 2.4 \\
\hline DCE-09-83-MB14 & 19.1 & 5.87 & 1721 & 196 & 1.95 & 444 & 6.96 & 26.50 & 55.3 & 0.05 & 610 & 2.3 & 95.9 & $<0.1$ & 470 & 14.8 & -5.4 \\
\hline & & 5.34 & 694 & 342 & & & & 17.40 & 10.2 & 0.32 & 120 & 1.2 & 31.1 & $<0.1$ & 330 & 23.3 & -5.5 \\
\hline DCE-09-85-MB16 & 20.4 & 5.14 & 155 & 308 & 0.78 & 45.1 & 0.98 & 1.93 & 1.99 & 0.06 & 30 & 1.3 & 25.5 & $<0.1$ & 46 & 22.1 & 2.3 \\
\hline DCE-09-86-MB17 & 20.2 & 5.07 & 199 & 362 & 2.40 & 57.0 & 0.61 & 3.92 & 2.82 & 0.05 & 38 & 1.3 & 14.2 & 0.2 & 80 & 26.9 & -0.6 \\
\hline & 22.3 & 6.04 & 860 & 302 & 2.26 & 217 & 4.49 & 31.30 & 16.5 & 0.68 & 240 & 2.1 & 127.7 & 0.7 & 220 & 14.3 & -3.9 \\
\hline DCE-C & 16.7 & 5.60 & 2197 & 340 & 3.72 & 600 & 14.1 & 61 & 49 & 0.18 & 1100 & 3.9 & 85.1 & 1.7 & 280 & 19.1 & -6.6 \\
\hline DCE-0 & 16.1 & 5.5 & 288 & 326 & 1.23 & 56.6 & 6.59 & 21.90 & 7.49 & 0.06 & 66 & 0.47 & 46.1 & $<0.1$ & 81 & 6.8 & 1.2 \\
\hline & 16.2 & 5.79 & 1005 & 330 & 0.39 & 180 & 11.0 & 81 & 38.1 & 0.02 & 310 & 1.6 & 43.0 & $<0.1$ & 340 & 9.1 & -4 \\
\hline 9-OS3 & 26.3 & 5.36 & 149 & 308 & 1.22 & 41.7 & 0.68 & 3.19 & 1.33 & 0.02 & 29 & 0.85 & 34.8 & $<0.1$ & 37 & 13.0 & -0.9 \\
\hline DCE-09-91-P1D & 28.2 & 5.37 & 6737 & 246 & 1.57 & 1820 & 7.98 & 44.7 & 331 & 0.20 & 4000 & 9.9 & 35.7 & $<0.1$ & 460 & 20.2 & -5.8 \\
\hline P1S & 20 & 3.8 & 3756 & 514 & 2.4 & 1130 & 7.56 & 9.32 & 95.2 & 0.05 & 2000 & 5.4 & 0.0 & $<0.1$ & 500 & 38.0 & -7.2 \\
\hline DCE-09-78-P2D & 23.1 & 5.82 & 2607 & 236 & 2.16 & 771 & 14.8 & 22.30 & 71.6 & 0.27 & 1310 & 3.8 & 109.2 & 0.2 & 300 & 17.9 & -4.7 \\
\hline DCE-09-95-CH17 & 21.7 & 3.86 & 2706 & 540 & 2.42 & 826 & 14.3 & 7.34 & 74.6 & 0.02 & 1600 & 7.1 & 0.0 & 10 & 160 & 41.0 & -6 \\
\hline & & 4.49 & 648 & 394 & & 200 & 5.48 & 1.15 & 5.59 & 0.05 & 140 & 8.7 & 5.2 & 0.3 & 280 & 43.7 & -2.7 \\
\hline DCE-09-97-CH21 & 18 & 4.57 & 534 & 387 & 6.00 & 156 & 4.24 & 0.70 & 2.64 & 0.03 & 52 & 2.2 & 15.2 & $<0.1$ & 300 & 48.1 & -5 \\
\hline DCE-09-98-CH30 & 18.6 & 4.29 & 333 & 495 & 4.58 & 97.0 & 1.25 & 1.06 & 3.22 & 0.04 & 39 & 1.6 & & $<0.1$ & 190 & 48.3 & -5.1 \\
\hline & & & 1528 & 296 & & & 14.0 & 11.20 & 20.9 & 0.13 & 460 & 6.1 & 154.5 & $<0.1$ & 410 & 24.1 & -4.4 \\
\hline DCE. & & $6 . c$ & 6258 & 78 & 2.8 & 98 & 457 & 64.7 & 109.0 & 0.76 & 1490 & 9.5 & 3129.6 & $<0.1$ & 3 & 14.8 & -8.9 \\
\hline DCE-09-101-MW13 & 19.7 & 4.60 & 4788 & 386 & 0.44 & 1590 & 21 & 10.80 & 122.0 & 0.44 & 2600 & 9.9 & & $<0.1$ & 430 & 29.3 & -1.2 \\
\hline DCE-09-103-MB301 & 20.8 & 6.61 & 1978 & 314 & $6 . c$ & 381 & 7.32 & 105 & 94.7 & 0.01 & 900 & 2.3 & 425.8 & 0.3 & 62 & 5.3 & -6.6 \\
\hline DCE-0 & & 5.49 & 178 & 126 & 1.6 & 49.7 & 4.20 & 2.35 & 2.53 & 0.03 & 43 & 0.50 & 32.6 & 0.4 & 41 & 22.5 & 0.8 \\
\hline DCE-09-107-D18 & 20.8 & 5.09 & 158 & 256 & 0.92 & 41.1 & 0.59 & 0.39 & 0.995 & 0.02 & 25 & 0.97 & 23.6 & 0.2 & 62 & 22.4 & -8.4 \\
\hline
\end{tabular}


Supplementary Paper S4 Groundwater and surface water information with well location, screened interval and abbreviated water type.

\begin{tabular}{|c|c|c|c|c|c|c|c|c|}
\hline & $\begin{array}{l}\text { Sample } \\
\text { Date }\end{array}$ & Northing & Easting & $\begin{array}{l}\text { Ground } \\
\text { Elevation } \\
\text { m (AHD) }\end{array}$ & $\begin{array}{c}\begin{array}{c}\text { Well } \\
\text { depth }\end{array} \\
\text { m (toc) }\end{array}$ & $\begin{array}{c}\text { Standing } \\
\text { water } \\
\text { level } \\
\text { m (toc) }\end{array}$ & $\begin{array}{c}\begin{array}{c}\text { Screen } \\
\text { depth }\end{array} \\
\begin{array}{c}\text { From to } m \\
\text { BGL }\end{array}\end{array}$ & Water type \\
\hline 2007 & & & & & & & & \\
\hline DCE-07-02-BH10 & $14 / 08 / 07$ & 6232117.6 & 313300.1 & 128.89 & 6.0 & 0.66 & $1.15-6.00$ & $\mathrm{Na}-\mathrm{Cl}$ \\
\hline DCE-07-01-BHF & $16 / 08 / 07$ & 6231996.0 & 313324.4 & & 11.7 & 1.33 & & \\
\hline DCE-07-19-CW & $15 / 08 / 07$ & 6232125.6 & 313213.1 & 129.51 & 12.5 & 3.89 & $5.9-11$ & $\mathrm{Na}-\mathrm{Cl}$ \\
\hline DCE-07-05-MB11 & $15 / 08 / 07$ & 6231816.4 & 313172.9 & & 7.4 & 3.97 & $1.00-$ & $\mathrm{Na}-\mathrm{Cl}$ \\
\hline DCE-07-06-MB12 & $16 / 08 / 07$ & 6231802.4 & 313268.1 & 129.05 & 4.8 & 2.90 & $0.60-476$ & $\mathrm{Na}-\mathrm{SO}_{4}$ \\
\hline DCE-07-07-MB13 & $14 / 08 / 07$ & 6231881.2 & 313279.8 & 129.50 & 5.9 & 1.21 & $0.30-5.90$ & \\
\hline DCE & $10 /$ & & & & 6.3 & 2.78 & & \\
\hline DCE-07-09-MB15 & $15 / 08 / 07$ & 6232014.3 & 313202.7 & & 6.8 & 3.34 & $0.45-6$. & $\mathrm{Na}-\mathrm{SO}_{4}$ \\
\hline DCE & $17 / 08 / 07$ & 6232004.1 & 71.7 & & 5.9 & 1.68 & 0.50 & \\
\hline DCE-07-11-MB17 & $14 / 08 / 07$ & 6232098.7 & 313312.2 & & 4.8 & 0.96 & 0.50 & \\
\hline DCE-07-12-MB18 & $15 / 08 / 07$ & 6232112.8 & 313216.9 & 129.99 & 5.9 & 3.34 & $0.40-5.86$ & $\mathrm{Na}-\mathrm{Cl}$ \\
\hline DCE-07-13-MB19 & $16 / 08 / 07$ & 6232112.6 & 313370.2 & 128.87 & 6.1 & 3.13 & $0.20-5.80$ & $\mathrm{Na}-\mathrm{Cl}$ \\
\hline DCE-07-14-MB20 & $16 / 08 / 07$ & 6231989.9 & 313362.0 & 131.52 & 5.1 & 0.79 & $0.45-4.80$ & \\
\hline 7-15-MB21 & $16 / 08 / 07$ & 623 & 51.3 & & 4.3 & 0.53 & 0.30 & \\
\hline $\mathrm{DC}$ & $8 / 07$ & 623 & 96.3 & & 1.3 & 0.70 & ope & \\
\hline $\mathrm{DCE}$ & 8/07 & 6232055.5 & 13.9 & & 3.9 & 0.76 & & \\
\hline DCE-07-17-P1D & $28 / 08 / 07$ & 6231839.4 & 313270.9 & 28.92 & 22.0 & 3.36 & 15.00 & \\
\hline DCE-07-16.P1S & $17 / 08 / 07$ & 6231834.3 & 313270.4 & 128.94 & 5.9 & 2.34 & $2.10-5.83$ & $\mathrm{Na}-\mathrm{Cl}$ \\
\hline $\begin{array}{c}\text { DCE-07-18-P2D } \\
\text { Mar-08 }\end{array}$ & $28 / 08 / 07$ & 6232162.1 & 313232.2 & 127.84 & 33.7 & 11.22 & $29.00-36.44$ & $\mathrm{Na}-\mathrm{Cl}$ \\
\hline DCE-08-14-BH10 & & & & & 6.0 & 0.76 & $1.15-6.00$ & \\
\hline DCE-08-33-BHA & $31 / 03 / 08$ & 6232155.4 & 313269.5 & 127.56 & 10.4 & 2.05 & & \\
\hline DCE-08-13-BHF & $28 / 03 / 08$ & 6231996.0 & 313324.4 & 131.79 & 11.7 & 1.93 & $0-11.60$ & $\mathrm{Na}-\mathrm{Cl}$ \\
\hline DCE-08-31-CW & $26 / 03 / 08$ & 6232125.6 & 313213.1 & 9.51 & 12.5 & 4.08 & & \\
\hline 3-17-MB11 & $26 / 0$ & 6231816.4 & 172.9 & 2.33 & 7.4 & 3.44 & $1.00-7.30$ & $\mathrm{Ja}-\mathrm{Cl}$ \\
\hline DCE-08-18-MB12 & $28 / 03 / 08$ & 6231802.4 & 313268.1 & .05 & 4.8 & 2.04 & $0.60-476$ & $\mathrm{Na}-\mathrm{SO}_{4}$ \\
\hline & $28 /($ & & 31 & & 5.9 & 1.40 & & \\
\hline $\mathrm{DC}$ & $26 / C$ & 623 & 313 & & 6.3 & 2.70 & 0.4 & \\
\hline $\mathrm{DCE}$ & $26 / 03 / 08$ & 6232014.3 & 313202.7 & 37 & 6.8 & 3.50 & 0.4 & $\mathrm{Na}-\mathrm{SO}_{4}$ \\
\hline & $28 / 0$ & 623 & 71.7 & & 5.9 & 1.95 & 0.5 & \\
\hline 08-23-MB17 & $27 / 0$ & 6232098.7 & 12.2 & & 4.8 & 1.06 & 0.50 & \\
\hline DCE-08-24-MB18 & $26 / 03 / 08$ & 6232112.8 & 313216.9 & & 5.9 & 3.34 & & \\
\hline DCE-08-25-MB19 & $31 / 03 / 08$ & 6232112.6 & 313370.2 & 128.87 & 6.1 & 2.16 & $0.20-5.80$ & \\
\hline DCE-08-26-MB20 & $31 / 03 / 08$ & 6231989.9 & 313362.0 & 131.52 & 5.1 & 1.55 & $0.45-4.80$ & $\mathrm{Na}-\mathrm{HCO}_{3}$ \\
\hline DCE-08-27-MB21 & $31 / 03 / 08$ & 6231910.0 & 313351.3 & 129.95 & 4.3 & 1.41 & $0.30-4.20$ & \\
\hline DCE-08-32-MB22 & $31 / 03 / 08$ & 6231792.6 & 313317.3 & 127.71 & 2.0 & 1.18 & $0.55-1.99$ & $\mathrm{Na}-\mathrm{Cl}$ \\
\hline DCE-08-15-OS2 & $28 / 03 / 08$ & 6231933.9 & 313296.3 & 131.05 & 1.3 & 0.99 & open base & $\mathrm{Na}-\mathrm{HCO}_{3}$ \\
\hline DCE-08-16-OS3 & $27 / 03 / 08$ & 6232055.5 & 313313.9 & 131.08 & 3.9 & 1.02 & open base & $\mathrm{Na}-\mathrm{HCO}_{3}$ \\
\hline DCE-08-28-P1D & $27 / 03 / 08$ & 6231839.4 & 313270.9 & 128.92 & 22.0 & 2.80 & $15.00-22.06$ & \\
\hline DCE-08-29-P1S & $28 / 03 / 08$ & 6231834.3 & 313270.4 & 128.94 & 5.9 & 2.22 & $2.10-5.83$ & $\mathrm{Na}-\mathrm{Cl}$ \\
\hline $\begin{array}{l}\text { DCE-08-30-P2D } \\
\text { Sep-Nov } 2008\end{array}$ & $27 / 03 / 08$ & 6232162.1 & 313232.2 & 127.84 & 33.7 & 13.15 & $29.00-36.44$ & $\mathrm{Na}-\mathrm{Cl}$ \\
\hline DCE-08-157-BH10 & & & & & 6.0 & 0.65 & & \\
\hline & & 6232155.4 & & & 10.4 & 2.54 & & \\
\hline DCE-08-176-BHB & $8 / 10 / 08$ & 6232147.3 & 313329.7 & 127.60 & 10.7 & 0.63 & $0-10.4$ & $\mathrm{Na}-\mathrm{Cl}$ \\
\hline DCE-08-156-BHF & $30 / 09 / 08$ & 6231996.0 & 313324.4 & 131.79 & 11.7 & 1.43 & $0-11.60$ & $\mathrm{Na}-\mathrm{Cl}$ \\
\hline DCE-08-174-CW & $26 / 09 / 08$ & 6232125.6 & 313213.1 & 129.51 & 12.5 & 4.06 & $5.9-11.4$ & $\mathrm{Na}-\mathrm{Cl}$ \\
\hline DCE-08-160-MB11 & $25 / 09 / 08$ & 6231816.4 & 313172.9 & 132.33 & 7.4 & 3.44 & $1.00-7.30$ & $\mathrm{Na}-\mathrm{SO}_{4}$ \\
\hline DCE-08-161-MB12 & 2/10/08 & 6231802.4 & 313268.1 & 129.05 & 4.8 & 1.75 & $0.60-476$ & $\mathrm{Na}-\mathrm{SO}_{4}$ \\
\hline DCE-08-162-MB13 & $1 / 10 / 08$ & 6231881.2 & 313279.8 & 129.50 & 5.9 & 1.20 & $0.30-5.90$ & $\mathrm{Na}-\mathrm{Cl}$ \\
\hline
\end{tabular}




\begin{tabular}{|c|c|c|c|c|c|c|c|c|}
\hline & $\begin{array}{c}\text { Sample } \\
\text { Date }\end{array}$ & Northing & Easting & $\begin{array}{l}\text { Ground } \\
\text { Elevation } \\
\text { m (AHD) }\end{array}$ & $\begin{array}{c}\text { Well } \\
\text { depth } \\
\text { m (toc) }\end{array}$ & $\begin{array}{l}\text { Standing } \\
\text { water } \\
\text { level } \\
\text { m (toc) }\end{array}$ & $\begin{array}{c}\text { Screen } \\
\text { depth } \\
\text { From to } m \\
\text { BGL }\end{array}$ & Water type \\
\hline DCE-08-163-MB14 & $26 / 09 / 08$ & 6231916.9 & 313188.6 & 132.54 & 6.3 & 2.58 & $0.45-6.25$ & $\mathrm{Na}-\mathrm{Cl}$ \\
\hline DCE-08-164-MB15 & $25 / 09 / 08$ & 6232014.3 & 313202.7 & 133.37 & 6.8 & 3.35 & $0.45-6.80$ & \\
\hline DCE-08-165-MB16 & $8 / 10 / 08$ & 6232004.1 & 313271.7 & 132.79 & 5.9 & 0.72 & 0.50 & $\mathrm{Na}-\mathrm{Cl}$ \\
\hline DCE-08-166-MB17 & $30 / 09 / 08$ & 6232098.7 & 313312.2 & 129.55 & 4.8 & 0.92 & $0.50-4.80$ & $\mathrm{Na}-\mathrm{SO}_{4}$ \\
\hline DCE-08-167-MB18 & $26 / 09 / 08$ & 6232112.8 & 313216.9 & 129.99 & 5.9 & 2.29 & $0.40-5.86$ & \\
\hline DCE-08-168-MB19 & $3 / 10 / 08$ & 6232112.6 & 313370.2 & 128.87 & 6.1 & 1.51 & 0.2 & \\
\hline DCE-08-169-MB20 & $7 / 10 / 08$ & 6231989.9 & 313362.0 & 131.52 & 5.1 & 0.27 & $0.45-4.80$ & $\mathrm{Na}-\mathrm{HCO}_{3}$ \\
\hline DCE-08-170-MB21 & $7 / 10 / 08$ & 6231910.0 & 313351.3 & 129.95 & 4.3 & 0.14 & $0.30-4.20$ & \\
\hline DCE-0 & $30 / 09 / 08$ & 6231 & 96.3 & & 1.3 & 0.74 & ope & \\
\hline DCE-08-159-OS3 & $30 / 09 / 08$ & 6232055.5 & 313 & & 3.9 & 0.77 & & \\
\hline DCE-08-171-P1D & 2/10/08 & 6231839.4 & 313270.9 & & 22.0 & 2.58 & $15.00-22.06$ & \\
\hline DCE-08-172-P1S & $1 / 10 / 08$ & 6231834.3 & 313270.4 & 128.94 & 5.9 & 1.94 & $2.10-5.83$ & \\
\hline DCE-08-173-P2D & $3 / 10 / 08$ & 6232162.1 & 313232.2 & 127.84 & 33.7 & 13.35 & $29.00-36.44$ & $\mathrm{Na}-\mathrm{Cl}$ \\
\hline DCE-08-177-D14 & $2 / 10 / 08$ & 6231988.1 & 313234.5 & 132.96 & 2.6 & 1.17 & $0-3.00$ & $\mathrm{Na}-\mathrm{SO}_{4}$ \\
\hline DCE-08-180-JTC & $8 / 10 / 08$ & 6231726.6 & 313411.1 & 5.84 & $\begin{array}{r}\text { surface } \\
\text { water }\end{array}$ & N/A & N/A & \\
\hline 2009 & & & & & & & & \\
\hline DCE-09-77-BH10 & $22 / 10 / 09$ & 6232117.6 & 313300.1 & 128.89 & 6.0 & 1.07 & & $\mathrm{Na}-\mathrm{Cl}$ \\
\hline DCE-09-76-BHF & $10 / 11 / 09$ & 96.0 & 24.4 & & 11.7 & 2.19 & & \\
\hline DCE & 9 & & & & 12.5 & 4. & & \\
\hline DCE & $23 /$ & 623 & 2.9 & & 7.4 & 3.95 & & \\
\hline DCE-C & $3 / 11 / 09$ & 802.4 & 313268.1 & .05 & 4.8 & 2.32 & 0.6 & $\mathrm{Na}-\mathrm{SO}_{4}$ \\
\hline DCE & & 623 & 9.8 & & 5.9 & 1.25 & & \\
\hline DCE-09-83 & $23 / 1$ & 6.9 & 88.6 & & 6.3 & 3.14 & & \\
\hline DCE-09-84-MB15 & $29 / 10 / 09$ & 6232014.3 & 313202.7 & 133.37 & 6.8 & 4.13 & $0.45-6.80$ & $\mathrm{Na}-\mathrm{SO}_{4}$ \\
\hline DCE-09-85-MB16 & 9/11/09 & 6232004.1 & 313271.7 & 132.79 & 5.9 & 2.37 & $0.50-5.90$ & $\mathrm{Na}-\mathrm{SO}_{4}$ \\
\hline DCE-09-86-MB17 & $22 / 10 / 09$ & 6232098.7 & 12.2 & .55 & 4.8 & 1.40 & 0.50 & $\mathrm{Na}-\mathrm{SO}_{4}$ \\
\hline DCE-0 & //10/09 & 6232 & 16.9 & & 5.9 & 3.96 & & \\
\hline & & & & & 6.1 & & & \\
\hline DCE-0 & & 623 & 62.0 & & 5.1 & 1.41 & & \\
\hline DCE-09-90-MB21 & & 6231 & 51.3 & & 4.3 & 1.60 & $0.30-4.20$ & \\
\hline DCE-09-79-OS3 & $10 / 11 / 09$ & 6232055.5 & 313313.9 & & 3.9 & 1.25 & open base & \\
\hline DCE-09-91-P1D & $12 / 11 / 09$ & 6231839.4 & 313270.9 & & 22.0 & 3.60 & $15.00-22.06$ & \\
\hline DCE-09-92-P1S & & 6231834.3 & 313270.4 & & 5.9 & 2.61 & & \\
\hline & & & & & 33.7 & 13.95 & 29.00 & \\
\hline & & & & & 4.2 & 1.24 & & \\
\hline DCE-09-96-CH18 & 22/10/09 & 6232107.3 & 313306.4 & 129.33 & 4.0 & 1.67 & 3.05-3.95 & $\mathrm{Na}-\mathrm{SO}_{4}$ \\
\hline DCE-09-97-CH21 & $27 / 10 / 09$ & 6232068.0 & 313298.9 & 130.53 & 5.4 & 1.87 & $3.42-4.32$ & $\mathrm{Na}-\mathrm{SO}_{4}$ \\
\hline DCE-09-98-CH30 & 28/10/09 & 6231966.8 & 313265.6 & 132.26 & 4.2 & 1.18 & $2.9-3.8$ & $\mathrm{Na}-\mathrm{SO}_{4}$ \\
\hline DCE-09-99-CH31 & 28/10/09 & 6231948.8 & 313248.9 & 131.94 & 7.0 & 1.94 & $5.65-6.7$ & $\mathrm{Na}-\mathrm{Cl}$ \\
\hline DCE-09-100-LHQ & $16 / 11 / 09$ & 6232257.0 & 313172.0 & 126.21 & 7.1 & 5.41 & $0-7.10$ & $\mathrm{Na}-\mathrm{HCO}_{3}$ \\
\hline DCE-09-101-MW13 & $30 / 10 / 09$ & 6232252.0 & 313214.0 & 125.30 & 5.5 & 3.63 & 2.50 to 5.50 & $\mathrm{Na}-\mathrm{Cl}$ \\
\hline DCE-09-103-MB301 & $30 / 10 / 09$ & 6231872.0 & 312859.0 & 137.00 & 26.5 & 22.01 & $19.00-25.00$ & $\mathrm{Na}-\mathrm{Cl}$ \\
\hline DCE-09-105-D14 & $12 / 11 / 09$ & 6231988.1 & 313234.5 & 132.96 & 2.6 & 1.60 & $0-3.00$ & $\mathrm{Na}-\mathrm{Cl}$ \\
\hline DCE-09-107-D18 & $13 / 11 / 09$ & 6232029.2 & 313265.1 & 132.53 & 3.1 & 1.80 & $0-3.00$ & $\mathrm{Na}-\mathrm{SO}_{4}$ \\
\hline
\end{tabular}


Supplementary Paper S5 Selected groundwater minor and trace element concentrations. Al, Mn and Fe analysed by ICP-AES, the rest of elements by ICP-MS. BDL (bellow detection limit); no data represent not analysed elements.

\begin{tabular}{|c|c|c|c|c|c|c|c|}
\hline & $\begin{array}{c}\mathrm{Be} \\
\mu \mathrm{g} \mathrm{L}^{-1}\end{array}$ & $\begin{array}{c}\text { Al } \\
\mu \mathrm{g} \mathrm{L}^{-1}\end{array}$ & $\begin{array}{c}\mathrm{Mn} \\
\mu \mathrm{g} \mathrm{L}^{-1}\end{array}$ & $\begin{array}{c}\mathrm{Fe} \\
\mu \mathrm{g} \mathrm{L}^{-1}\end{array}$ & $\begin{array}{c}\mathrm{Sr} \\
\mu \mathrm{gL}^{-1}\end{array}$ & $\begin{array}{c}\mathrm{I} \\
\mu \mathrm{g} \mathrm{L}^{-1}\end{array}$ & $\begin{array}{c}\mathbf{U} \\
\mu \mathrm{gL}^{-1}\end{array}$ \\
\hline \multicolumn{8}{|l|}{ Aug-07 } \\
\hline DCE-07-02-BH10 & 0.188 & 64.1 & 0.184 & 0.09 & 0.003 & 47 & 0.053 \\
\hline DCE-07-01-BHF & 0.260 & 237.5 & 1.120 & 1.63 & 0.078 & 174 & 0.074 \\
\hline DCE-07-19-CW & 12.174 & 50.2 & 1.530 & 0.07 & 0.074 & 493 & 0.053 \\
\hline DCE-07-05-MB11 & 0.132 & 20.9 & 0.296 & 0.05 & 0.047 & 5 & 0.013 \\
\hline DCE-07-06-MB12 & 0.089 & 36.3 & 0.081 & 0.04 & 0.054 & 31 & 0.08 \\
\hline DCE-07-07-MB13 & 0.062 & 54.5 & 0.081 & 0.31 & 0.034 & 9 & 0.049 \\
\hline DCE-07-08-MB14 & 0.376 & 89.0 & 0.681 & 7.32 & 0.189 & 283 & 0.013 \\
\hline DCE-07-09-MB15 & 10.909 & 197.8 & 1.180 & 0.70 & 0.125 & 608 & 0.024 \\
\hline DCE-07-10-MB16 & 0.050 & 26.9 & 0.087 & 1.92 & 0.041 & 3180 & 0.051 \\
\hline DCE-07-11-MB17 & 0.025 & 40.0 & 0.060 & 0.05 & 0.03 & 168 & 0.099 \\
\hline DCE-07-12-MB18 & 0.964 & 15.3 & 0.122 & 0.02 & 0.331 & 250 & 0.021 \\
\hline DCE-07-13-MB19 & 0.106 & 14.2 & 7.790 & 2.40 & 1.522 & 125 & 0.062 \\
\hline DCE-07-14-MB20 & 0.031 & 109.6 & 0.493 & 0.87 & 0.212 & 221 & 0.049 \\
\hline DCE-07-15-MB21 & 0.009 & 44.2 & 0.082 & 0.08 & 0.115 & 61 & 0.042 \\
\hline DCE-07-03-OS2 & 0.005 & 28.1 & 0.002 & 0.06 & 0.04 & 22 & 0.044 \\
\hline DCE-07-04-OS3 & 0.018 & 16.1 & 0.015 & 0.17 & 0.009 & 465 & 0.074 \\
\hline DCE-07-17-P1D & 17.517 & 81.2 & 27.100 & 145 & 0.361 & 678 & 0.093 \\
\hline DCE-07-16.P1S & 8.905 & 5112.3 & 6.470 & 8.21 & 0.117 & 338 & 1.765 \\
\hline DCE-07-18-P2D & 3.871 & 1098.1 & 4.230 & 17.00 & 0.368 & 369 & 0.204 \\
\hline \multicolumn{8}{|l|}{ Mar-08 } \\
\hline DCE-08-14-BH10 & 0.212 & 86.5 & 0.530 & 0.25 & 0.009 & 660 & 0.1 \\
\hline DCE-08-33-BHA & 0.014 & 4.9 & 1.500 & 10.60 & 0.329 & 190 & 0.362 \\
\hline DCE-08-13-BHF & 0.246 & 81.2 & 2.530 & 4.02 & 0.059 & 1140 & 0.05 \\
\hline DCE-08-31-CW & 11.622 & 50.5 & 2.000 & 0.13 & 0.089 & 650 & 0.083 \\
\hline DCE-08-17-MB11 & 0.175 & 40.6 & 0.600 & 0.11 & 0.058 & 20 & 0.02 \\
\hline DCE-08-18-MB12 & 0.092 & 39.1 & 0.200 & 0.03 & 0.064 & 90 & 0.056 \\
\hline DCE-08-19-MB13 & 0.114 & 75.1 & 0.200 & 0.15 & 0.093 & 150 & 0.043 \\
\hline DCE-08-20-MB14 & 0.313 & 90.1 & 0.500 & 0.16 & 0.269 & 250 & 0.019 \\
\hline DCE-08-21-MB15 & 10.410 & 188.6 & 0.200 & 0.13 & 0.121 & 580 & 0.022 \\
\hline DCE-08-22-MB16 & 0.052 & 39.6 & 0.100 & 0.34 & 0.048 & 1680 & 0.055 \\
\hline DCE-08-23-MB17 & 0.053 & 34.6 & $<0.1$ & 0.40 & 0.039 & 1380 & 0.125 \\
\hline DCE-08-24-MB18 & 5.839 & 91.0 & 1.600 & 0.05 & 0.548 & 500 & 0.032 \\
\hline DCE-08-25-MB19 & 0.323 & 17.1 & 6.100 & 2.07 & 1.134 & 170 & 0.095 \\
\hline DCE-08-26-MB20 & 0.036 & 38.4 & 1.100 & 3.49 & 0.449 & 330 & 0.07 \\
\hline DCE-08-27-MB21 & 0.016 & 14.0 & 1.600 & 0.24 & 0.983 & 730 & 0.056 \\
\hline DCE-08-32-MB22 & 0.088 & 149.8 & 0.500 & 4.18 & 0.191 & 70 & 0.224 \\
\hline DCE-08-15-OS2 & 0.004 & 12.6 & $<0.1$ & 0.07 & 0.065 & 90 & 0.057 \\
\hline DCE-08-16-OS3 & 0.020 & 29.1 & $<0.1$ & 17.90 & 0.039 & 6980 & 0.309 \\
\hline DCE-08-28-P1D & 29.285 & 181.0 & 22.200 & 122.00 & 0.354 & 670 & 0.265 \\
\hline DCE-08-29-P1S & 9.027 & 4540.4 & 6.800 & 9.81 & 0.12 & 710 & 1.848 \\
\hline DCE-08-30-P2D & 1.345 & 94.8 & 2.400 & 15.80 & 0.161 & 240 & 0.07 \\
\hline \multicolumn{8}{|l|}{ Sep-Nov 2008} \\
\hline DCE-08-157-BH10 & 0.246 & 84.7 & 1.460 & 3.87 & 0.011 & 1100 & 0.048 \\
\hline DCE-08-175-BHA & 0.027 & 3.2 & 1.350 & 7.56 & 0.241 & 210 & 0.485 \\
\hline DCE-08-176-BHB & 0.022 & 1.7 & 4.710 & 61.90 & 0.700 & 140 & \\
\hline
\end{tabular}




\begin{tabular}{|c|c|c|c|c|c|c|c|}
\hline & $\begin{array}{c}\mathrm{Be} \\
\mu \mathrm{g} \mathrm{L}^{-1}\end{array}$ & $\begin{array}{c}\text { Al } \\
\mu \mathrm{g} \mathrm{L}^{-1}\end{array}$ & $\begin{array}{c}\mathrm{Mn} \\
\mu \mathrm{g} \mathrm{L}^{-1}\end{array}$ & $\begin{array}{c}\mathrm{Fe} \\
\mu \mathrm{g} \mathrm{L}^{-1}\end{array}$ & $\begin{array}{c}\mathrm{Sr} \\
\mu \mathrm{g} \mathrm{L}^{-1}\end{array}$ & $\begin{array}{c}\mathrm{I} \\
\mu \mathrm{g} \mathrm{L}^{-1}\end{array}$ & $\underset{\mu \mathrm{g} \mathrm{L}^{-1}}{\mathrm{U}}$ \\
\hline DCE-08-156-BHF & 0.382 & 140.9 & 1.950 & 1.34 & 0.082 & 770 & 0.03 \\
\hline DCE-08-174-CW & 12.743 & 40.2 & 1.960 & 0.395 & 0.091 & 640 & 0.047 \\
\hline DCE-08-160-MB11 & 0.086 & 27.9 & 0.164 & 0.061 & 0.026 & $<10$ & 0.025 \\
\hline DCE-08-161-MB12 & 0.092 & 57.8 & 0.124 & 0.01 & 0.072 & 70 & 0.055 \\
\hline DCE-08-162-MB13 & 0.094 & 89.5 & 0.153 & 0.146 & 0.066 & 90 & 0.054 \\
\hline DCE-08-163-MB14 & 0.278 & 68.8 & 0.368 & 3.19 & 0.243 & 80 & 0.014 \\
\hline DCE-08-164-MB15 & 0.652 & 39.3 & 0.355 & 0.078 & 0.168 & 270 & 0.033 \\
\hline DCE-08-165-MB16 & 0.061 & 41.0 & 0.077 & 0.367 & 0.033 & 1330 & 0.056 \\
\hline DCE-08-166-MB17 & 0.045 & 34.7 & 0.131 & 0.092 & 0.041 & 1500 & 0.07 \\
\hline DCE-08-167-MB18 & 0.045 & 2.8 & 0.033 & 0.003 & 0.116 & 10 & 0.014 \\
\hline DCE-08-168-MB19 & 0.294 & 16.7 & 6.920 & 2.91 & 0.857 & 130 & 0.061 \\
\hline DCE-08-169-MB20 & 0.035 & 119.7 & 0.522 & 0.553 & 0.146 & 130 & 0.07 \\
\hline DCE-08-170-MB21 & 0.013 & 122.0 & 0.106 & 0.232 & 0.075 & 50 & 0.063 \\
\hline DCE-08-158-OS2 & 0.010 & 21.2 & 0.008 & 0.04 & 0.038 & 20 & 0.035 \\
\hline DCE-08-159-OS3 & 0.028 & 12.5 & 0.016 & 0.113 & 0.008 & 840 & 0.082 \\
\hline DCE-08-171-P1D & 18.172 & 45.8 & 20.600 & 122.0 & 0.355 & 670 & 0.033 \\
\hline DCE-08-172-P1S & 7.674 & 4144.2 & 8.080 & 11.10 & 0.106 & 1080 & 1.427 \\
\hline DCE-08-173-P2D & 1.577 & 75.2 & 2.970 & 24.3 & 0.192 & 310 & 0.075 \\
\hline DCE-08-177-D14 & bdl & bdl & 0.016 & 0.705 & bdl & 3890 & bdl \\
\hline DCE-08-180-JTC & 0.335 & 404.1 & 0.812 & 0.269 & 0.068 & 40 & 0.032 \\
\hline \multicolumn{8}{|l|}{ Oct-Nov 2009} \\
\hline DCE-09-77-BH10 & & 40.0 & 0.368 & 0.599 & 0.003 & 250 & \\
\hline DCE-09-76-BHF & & 120.0 & 6.860 & 3.24 & 0.095 & 650 & \\
\hline DCE-09-94-CW & & 40.0 & 2.020 & 0.128 & 0.075 & 710 & \\
\hline DCE-09-80-MB11 & & 40.0 & 0.674 & 0.018 & 0.063 & 20 & \\
\hline DCE-09-81-MB12 & & 60.0 & 0.200 & 0.03 & 0.065 & 160 & \\
\hline DCE-09-82-MB-13 & & 40.0 & 0.069 & 0.053 & 0.025 & 50 & \\
\hline DCE-09-83-MB14 & & 40.0 & 2.680 & 25.9 & 0.490 & 2070 & \\
\hline DCE-09-84-MB15 & & 40.0 & 1.130 & 0.27 & 0.214 & 1020 & \\
\hline DCE-09-85-MB16 & & bdl & 0.058 & 2.30 & 0.023 & 2080 & \\
\hline DCE-09-86-MB17 & & bdl & 0.127 & 0.37 & 0.043 & 1050 & \\
\hline DCE-09-87-MB18 & & 50.0 & 0.923 & 0.015 & 0.379 & 230 & \\
\hline DCE-09-88-MB19 & & 60.0 & 10.400 & 1.50 & 0.619 & 160 & \\
\hline DCE-09-89-MB20 & & bdl & 0.778 & 2.20 & 0.230 & 190 & \\
\hline DCE-09-90-MB21 & & bdl & 1.810 & 0.114 & 0.978 & 450 & \\
\hline DCE-09-79-OS3 & & bdl & 0.027 & 1.10 & 0.009 & 1690 & \\
\hline DCE-09-91-P1D & & bdl & 23.500 & 139.0 & 0.299 & 270 & \\
\hline DCE-09-92-P1S & & 3650.0 & 7.740 & 8.76 & 0.096 & 710 & \\
\hline DCE-09-78-P2D & & 30.0 & 3.460 & 28.7 & 0.199 & 230 & \\
\hline DCE-09-95-CH17 & & 5390.0 & 6.860 & 12.5 & 0.092 & 760 & \\
\hline DCE-09-96-CH18 & & 230.0 & 0.164 & 1.57 & 0.014 & 9700 & \\
\hline DCE-09-97-CH21 & & 100.0 & 0.075 & 1.26 & 0.007 & 9000 & \\
\hline DCE-09-98-CH30 & & 200.0 & 0.019 & 0.488 & 0.007 & 7100 & \\
\hline DCE-09-99-CH31 & & 70.0 & 4.620 & 24.2 & 0.095 & 27100 & \\
\hline DCE-09-100-LHQ & & 40.0 & 0.182 & 11.0 & 0.531 & 5500 & \\
\hline DCE-09-101-MW13 & & 1970.0 & 3.380 & 23.4 & 0.173 & 12200 & \\
\hline DCE-09-103-MB301 & & bdl & 0.136 & 0.018 & 0.379 & 280 & \\
\hline DCE-09-105-D14 & & 70.0 & 0.050 & 2.31 & 0.029 & 2260 & \\
\hline DCE-09-107-D18 & & bdl & 0.019 & 3.22 & 0.004 & 1540 & \\
\hline
\end{tabular}


Supplementary Paper $\mathbf{S 6}$ Groundwater dissolved organic carbon (DOC), stable isotopes $\left(\delta^{13} \mathrm{C}_{\mathrm{DOC}}, \delta^{13} \mathrm{C}_{\mathrm{DIC}}\right.$, water $\delta^{18} \mathrm{O}$ and $\delta^{2} \mathrm{H}$, sulfated $\delta^{34} \mathrm{~S}$ and $\delta^{18} \mathrm{O}$ ), calculated deuterium excess ( $d$-excess), ${ }^{87} \mathrm{Sr} /{ }^{86} \mathrm{Sr}$ and tritium $\left({ }^{3} \mathrm{H}\right.$ ). Note ${ }^{3} \mathrm{H}$ is resorted in $\mathrm{Ba} \mathrm{L}^{-1}\left(1 \mathrm{Ba} \mathrm{L}^{-1}=8.47 \mathrm{TU}\right)$.

\begin{tabular}{|c|c|c|c|c|c|c|c|c|c|c|}
\hline & DOC & $\delta^{13} C_{D O C}$ & $\delta^{13} C_{D I C}$ & $\delta^{18} \mathrm{O}$ & $\delta^{2} \mathrm{H}$ & d-excess & $\delta^{34} S_{\mathrm{SO} 4}$ & $\delta^{18} \mathrm{O}_{\mathrm{sO} 4}$ & ${ }^{87} \mathrm{Sr} /{ }^{86} \mathrm{Sr}$ & ${ }^{3} \mathrm{H}$ \\
\hline & \multirow[t]{2}{*}{$\mathrm{mg} \mathrm{L}^{-1}$} & $\%$ VPDB & $\%$ VPDB & $\%$ VSMOW\% & vsmow & $\%$ VSMOW & $\%$ VCDT & \%o VSMOW & & $\mathrm{Bq} \mathrm{L}^{-1}$ \\
\hline \multicolumn{10}{|l|}{2007} & \\
\hline DCE-07-02-BH10 & 6.28 & -18.3 & -19.4 & -4.61 & -23.4 & 13.5 & & & & 159 \\
\hline DCE-07-01-BHF & 8.03 & -19.3 & -19.8 & -4.86 & -24.4 & 14.5 & & & & 152 \\
\hline DCE-07-19-CW & 40.49 & -11.3 & -26.9 & -4.68 & -23.5 & 14.0 & & & & 710 \\
\hline DCE-07-05-MB11 & 3.84 & -20.3 & -28.5 & -4.78 & -22.7 & 15.5 & & & & 3 \\
\hline DCE-07-06-MB12 & 17.91 & -21.7 & -26.2 & -4.53 & -21.5 & 14.8 & & & & 3 \\
\hline DCE-07-07-MB13 & 7.84 & -14.2 & -26.3 & -4.51 & -19.8 & 16.3 & & & & 470 \\
\hline DCE-07-08-MB14 & 7.15 & -21.3 & -26.7 & -4.97 & -24.1 & 15.6 & & & & 34 \\
\hline DCE-07-09-MB15 & 17.26 & -20.2 & -20.2 & -4.80 & -26.0 & 12.4 & & & & 105 \\
\hline DCE-07-10-MB16 & 48.42 & -25.8 & -10.9 & -4.91 & -22.5 & 16.8 & & & & 2310 \\
\hline DCE-07-11-MB17 & 7.99 & -26.2 & -15.3 & -4.69 & -21.3 & 16.3 & & & & 196 \\
\hline DCE-07-12-MB18 & 8.53 & -16.3 & -26.4 & -4.73 & -23.2 & 14.6 & & & & 85 \\
\hline DCE-07-13-MB19 & 9.03 & -21.2 & -18.9 & -5.41 & -27.8 & 15.5 & & & & 402 \\
\hline DCE-07-14-MB20 & 8.12 & -27.2 & -12.0 & -4.75 & -19.5 & 18.5 & & & & 3 \\
\hline DCE-07-15-MB21 & 6.54 & -16.5 & -24.3 & -5.15 & -22.9 & 18.3 & & & & 14 \\
\hline DCE-07-03-OS2 & 6.60 & -14.6 & -25.1 & -4.52 & -21.8 & 14.4 & & & & 342 \\
\hline DCE-07-04-OS3 & 6.57 & -11.1 & -11.0 & -4.88 & -24.7 & 14.4 & & & & 291 \\
\hline DCE-07-17-P1D & 29.65 & -16.5 & -29.1 & -4.87 & -25.5 & 13.5 & & & & 125 \\
\hline DCE-07-16.P1S & 18.01 & -21.5 & -27.0 & -4.64 & -23.3 & 13.8 & & & & 41 \\
\hline DCE-07-18-P2D & 26.05 & -17.9 & -25.3 & -4.83 & -25.1 & 13.5 & & & & 108 \\
\hline \multicolumn{11}{|l|}{ Mar-08 } \\
\hline DCE-08-14-BH10 & 2.02 & -22.7 & -18.6 & -4.20 & -20.1 & 13.6 & 22.7 & 4.6 & 0.710968 & 477 \\
\hline DCE-08-33-BHA & 14.79 & -24.6 & -16.8 & -5.23 & -26.0 & 15.8 & 27.6 & 9.4 & & 52 \\
\hline DCE-08-13-BHF & 3.51 & -24.0 & -19.8 & -4.97 & -25.5 & 14.3 & 21.7 & 8.9 & 0.710983 & 643 \\
\hline DCE-08-31-CW & 6.10 & -23.8 & -11.1 & -4.76 & -23.5 & 14.6 & 21.1 & 14.7 & 0.709519 & 543 \\
\hline DCE-08-17-MB11 & 0.15 & -25.6 & -18.8 & -4.84 & -24.8 & 13.9 & 20.9 & 11.5 & & 0 \\
\hline DCE-08-18-MB12 & 0.17 & -25.3 & -21.5 & -4.37 & -20.3 & 14.7 & 22.0 & 13.8 & & 1 \\
\hline DCE-08-19-MB13 & 0.67 & -23.4 & -14.9 & -4.24 & -20.3 & 13.7 & 21.2 & 15.2 & & 501 \\
\hline DCE-08-20-MB14 & 0.46 & -25.5 & -21.2 & -4.96 & -25.0 & 14.7 & 23.1 & 12.5 & & 22 \\
\hline DCE-08-21-MB15 & 0.55 & -25.0 & -20.4 & -4.55 & -24.0 & 12.4 & 21.0 & 14.4 & & 13 \\
\hline DCE-08-22-MB16 & 0.68 & -24.5 & -13.2 & -4.94 & -23.2 & 16.3 & 21.7 & 13.4 & & 1589 \\
\hline DCE-08-23-MB17 & 6.15 & -23.3 & -15.6 & -4.51 & -21.7 & 14.4 & & & & 272 \\
\hline DCE-08-24-MB18 & 6.75 & -23.3 & -13.3 & -4.77 & -23.8 & 14.3 & & & & 1318 \\
\hline DCE-08-25-MB19 & 10.98 & -22.2 & -19.5 & -5.09 & -28.0 & 12.8 & 23.1 & 14.1 & & 361 \\
\hline DCE-08-26-MB20 & 20.76 & -18.7 & -10.5 & -4.80 & -23.5 & 14.9 & 21.0 & 15.4 & & 0 \\
\hline DCE-08-27-MB21 & 12.61 & -23.1 & -17.2 & -4.37 & -21.5 & 13.5 & 21.7 & 13.2 & & 260 \\
\hline DCE-08-32-MB22 & 13.07 & -25.4 & -22.1 & -4.79 & -26.2 & 12.1 & & & & 0 \\
\hline DCE-08-15-OS2 & 1.20 & -23.8 & -13.6 & -4.15 & -19.7 & 13.6 & & & & 273 \\
\hline DCE-08-16-OS3 & 2.52 & -23.2 & -9.0 & -4.61 & -21.7 & 15.2 & 24.7 & 9.4 & 0.711145 & 663 \\
\hline DCE-08-28-P1D & 7.44 & -25.8 & -17.6 & -4.74 & -26.2 & 11.7 & 21.2 & 14.5 & 0.711138 & 118 \\
\hline DCE-08-29-P1S & 8.51 & -24.6 & -21.6 & -4.65 & -25.1 & 12.1 & 23.1 & 15.1 & 0.711026 & 29 \\
\hline DCE-08-30-P2D & 7.68 & -25.0 & -18.2 & -4.98 & -27.9 & 12.0 & 20.0 & 13.3 & 0.710189 & 87 \\
\hline \multicolumn{11}{|l|}{ Sep-Nov 2008} \\
\hline DCE-08-157-BH10 & & & -19.1 & -4.68 & -21.3 & 16.2 & & & & 1924 \\
\hline DCE-08-175-BHA & & & -11.4 & -5.31 & -28.1 & 14.4 & & & & 53 \\
\hline DCE-08-176-BHB & & & -13.5 & -5.30 & -29.5 & 12.9 & & & & 59 \\
\hline DCE-08-156-BHF & & & -21.9 & -4.93 & -22.0 & 17.4 & & & 0.711066 & 514 \\
\hline DCE-08-174-CW & & & -11.4 & -5.14 & -26.0 & 15.1 & & & & 593 \\
\hline DCE-08-160-MB11 & & & -22.1 & -4.95 & -24.8 & 14.8 & & & & \\
\hline
\end{tabular}


Cendón et al. 2015 Supplementary Papers

Australian Journal of Earth Sciences 62, 123-141. http://dx.doi.org/10.1080/08120099.2015.975155

\begin{tabular}{|c|c|c|c|c|c|c|c|c|c|c|}
\hline & \multirow{2}{*}{$\begin{array}{l}\mathrm{DOC} \\
\mathrm{mg} \mathrm{L}^{-1}\end{array}$} & \multirow{2}{*}{$\begin{array}{c}\delta^{13} C_{D O C} \\
\% \text { VPDB }\end{array}$} & \multirow{2}{*}{$\begin{array}{c}\delta^{13} C_{\text {DIC }} \\
\% \text { VPDB }\end{array}$} & \multirow{2}{*}{$\begin{array}{c}\delta^{18} \mathrm{O} \\
\% \text { VSMOW\% }\end{array}$} & \multirow{2}{*}{$\begin{array}{c}\delta^{2} \mathrm{H} \\
\text { VSMOW }\end{array}$} & \multirow{2}{*}{$\begin{array}{l}\text { d-excess } \\
\% \text { VSMOW }\end{array}$} & \multirow{2}{*}{$\begin{array}{c}\delta^{34} \mathrm{~S}_{\mathrm{SO4}} \\
\% \circ \mathrm{VCDT}\end{array}$} & \multirow{2}{*}{$\begin{array}{c}\delta^{18} \mathrm{O}_{\text {so4 }} \\
\% \text { VSMOW }\end{array}$} & ${ }^{87} \mathrm{Sr} /{ }^{86} \mathrm{Sr}$ & \multirow{2}{*}{$\begin{array}{c}{ }^{3} \mathrm{H} \\
\mathrm{Bq} \mathrm{L}^{-1}\end{array}$} \\
\hline & & & & & & & & & & \\
\hline DCE-08-161-MB12 & & & -21.9 & -4.59 & -22.0 & 14.7 & & & & 2 \\
\hline DCE-08-162-MB13 & & & -15.2 & -4.43 & -21.9 & 13.6 & & & & 457 \\
\hline DCE-08-163-MB14 & & & -22.6 & -4.85 & -23.6 & 15.3 & & & & 12 \\
\hline DCE-08-164-MB15 & & & -21.6 & -4.55 & -23.2 & 13.2 & & & & 26 \\
\hline DCE-08-165-MB16 & & & -15.4 & -5.14 & -25.5 & 15.6 & & & & 1363 \\
\hline DCE-08-166-MB17 & & & -18.8 & -4.74 & -23.3 & 14.6 & & & & 397 \\
\hline DCE-08-167-MB18 & & & -18.0 & -4.63 & -22.5 & 14.5 & & & & 30 \\
\hline DCE-08-168-MB19 & & & -21.2 & -5.21 & -28.3 & 13.4 & & & & 321 \\
\hline DCE-08-169-MB20 & & & -10.7 & -4.15 & -18.3 & 14.9 & & & & 11 \\
\hline DCE-08-170-MB21 & & & -17.1 & -4.18 & -18.1 & 15.3 & & & & 15 \\
\hline DCE-08-158-OS2 & & & -14.5 & -4.42 & -21.7 & 13.7 & & & & 335 \\
\hline DCE-08-159-OS3 & & & -13.9 & -4.85 & -24.0 & 14.9 & & & & 317 \\
\hline DCE-08-171-P1D & & & -18.3 & -5.09 & -27.7 & 13.0 & & & & 102 \\
\hline DCE-08-172-P1S & & & -23.2 & -5.02 & -27.1 & 13.1 & & & & 33 \\
\hline DCE-08-173-P2D & & & -18.8 & -5.23 & -29.4 & 12.5 & & & & 101 \\
\hline DCE-08-177-D14 & & & -20.8 & -4.83 & -22.3 & 16.4 & & & & 451 \\
\hline DCE-08-180-JTC & & & -22.5 & -4.26 & -19.8 & 14.3 & & & 0.711025 & 25 \\
\hline \multicolumn{11}{|l|}{2009} \\
\hline DCE-09-77-BH10 & & & -19.0 & -4.08 & -16.6 & 16.1 & & & & 275.5 \\
\hline DCE-09-76-BHF & & & -20.6 & -4.63 & -21.9 & 15.2 & 21.5 & 13.7 & & 1833 \\
\hline DCE-09-94-CW & & & -11.2 & -5.13 & -23.3 & 17.8 & & & & 1256 \\
\hline DCE-09-80-MB11 & & & -19.6 & -4.55 & -21.1 & 15.3 & & & & 2 \\
\hline DCE-09-81-MB12 & & & -22.7 & -4.43 & -20.5 & 14.9 & 21.5 & 16.8 & & 0 \\
\hline DCE-09-82-MB-13 & & & -15.0 & -4.02 & -16.0 & 16.2 & 20.9 & 15.1 & & 355 \\
\hline DCE-09-83-MB14 & & & -21.6 & -5.06 & -26.1 & 14.3 & 22.1 & 13.9 & & 137 \\
\hline DCE-09-84-MB15 & & & -21.4 & -4.93 & -25.3 & 14.1 & 21.5 & 15.4 & & 211 \\
\hline DCE-09-85-MB16 & & & -12.2 & -5.05 & -22.9 & 17.5 & 21.8 & 13.9 & & 3290 \\
\hline DCE-09-86-MB17 & & & -18.4 & -4.46 & -20.6 & 15.1 & & & & 489 \\
\hline DCE-09-87-MB18 & & & -16.4 & -4.61 & -23.1 & 13.8 & & & & 2059 \\
\hline DCE-09-88-MB19 & & & -21.5 & -5.33 & -28.8 & 13.8 & 21.7 & 15.3 & & 572 \\
\hline DCE-09-89-MB20 & & & -11.9 & -3.97 & -16.1 & 15.7 & 20.1 & 15.1 & & 4 \\
\hline DCE-09-90-MB21 & & & -18.5 & -4.09 & -17.1 & 15.7 & 20.6 & 13.2 & & 279 \\
\hline DCE-09-79-OS3 & & & -11.5 & -4.61 & -20.7 & 16.2 & & & & 476 \\
\hline DCE-09-91-P1D & & & -18.4 & -4.99 & -28.8 & 11.2 & 21.4 & 14.4 & & 109 \\
\hline DCE-09-92-P1S & & & -23.0 & -5.11 & -26.6 & 14.3 & 22.6 & 16.7 & & 37 \\
\hline DCE-09-78-P2D & & & -18.7 & -5.04 & -26.7 & 13.6 & 20.6 & 14.7 & & 91 \\
\hline DCE-09-95-CH17 & & & -22.5 & -5.38 & -30.0 & 13.0 & 21.5 & 13.3 & 0.71648 & 14 \\
\hline DCE-09-96-CH18 & & & -19.5 & -5.24 & -22.8 & 19.2 & & & 0.71580 & 2585 \\
\hline DCE-09-97-CH21 & & & -14.5 & -5.38 & -18.4 & 24.7 & 21.3 & 16.3 & 0.71752 & 12165 \\
\hline DCE-09-98-CH30 & & & -13.5 & -5.05 & -15.8 & 24.6 & 21.1 & 14.4 & 0.71433 & 12489 \\
\hline DCE-09-99-CH31 & & & -16.1 & -4.78 & -20.9 & 17.3 & 21.5 & 13.8 & 0.71347 & 8287 \\
\hline DCE-09-100-LHQ & & & & -4.85 & 3.5 & 42.4 & & & & 267 \\
\hline DCE-09-101-MW13 & & & -1.9 & -5.08 & -17.0 & 23.7 & 21.9 & 14.4 & 0.71159 & 22 \\
\hline DCE-09-103-MB301 & & & -11.9 & -4.84 & -25.8 & 13.0 & 19.3 & 11.7 & & 8 \\
\hline DCE-09-105-D14 & & & -18.3 & -4.22 & -19.0 & 14.8 & 21.4 & 13.8 & & 696 \\
\hline DCE-09-107-D18 & & & -14.7 & -4.82 & -23.3 & 15.3 & 21.5 & 15.2 & & 366 \\
\hline
\end{tabular}


Supplementary Paper S7 Water stable isotopes for leachate samples from Harrington's Quarry. The $d$ is the calculated deuterium excess for the corresponding samples.

\begin{tabular}{l|ccc}
\hline & $\begin{array}{c}\delta^{18} \mathbf{0} \% \\
\text { VSMOW }\end{array}$ & $\begin{array}{c}\delta^{2} \mathrm{H} \% \\
\text { VSMOW }\end{array}$ & d-excess \\
\hline CEH-08-056 & & & \\
JJH-08-017 & -5.19 & 1.0 & 42.5 \\
JJH-08-019 & -5.00 & 2.5 & 42.5 \\
JJH-08-020 & -4.89 & 4.4 & 43.5 \\
JJH-08-023 & -4.95 & 11.6 & 51.1 \\
JJH-09-001 & -4.95 & 1.8 & 41.3 \\
JJH-09-007 & -5.36 & 15.7 & 58.6 \\
DCE-09-100-LHQ & -5.31 & 14.6 & 57.1 \\
\hline
\end{tabular}


Supplementary Paper S8 Calculated saturation indexes for common mineral phases in LFLS groundwater . Cal $=$ Calcite, Dol $=$ Dolomite, Sid $=$ Siderite, Gyp $=$ Gypsum, Hem $=$ Hematite, Goe $=$ Goethite, $\mathrm{Qtz}=$ Quartz, Bar = Barite, Cha $=$ Chalcedony, $\mathrm{Hal}=$ Halite, Illi $=$ Illite, $\mathrm{Kao}=$ Kaolinite.

\begin{tabular}{|c|c|c|c|c|c|c|c|c|c|c|c|c|}
\hline & si_Cal & si_Dol & si_Sid & si_Gyp & Hem s & si_Goe & si_Qtz & si_Bar & si_Cha & si_Hal & si_Illi & si_Kao \\
\hline DCE-07-02-BH10 & -5.27 & -10.15 & -3.89 & -4.11 & 4.38 & 1.21 & 0.20 & -1.46 & -0.26 & -6.98 & -3.85 & 2.02 \\
\hline CE-07-01-BHF & -4.98 & -9.10 & -2.98 & -3.57 & 45 & -0.76 & -0.05 & -0.45 & -0.51 & -6.26 & -2.63 & \\
\hline W & .09 & -5.47 & -2.95 & -2 & & -0 . & & -0 & & -5.17 & & -0.4 \\
\hline B11 & -4.92 & -9.25 & -4.47 & -3.29 & & 0.43 & & -0.42 & -0.28 & -7.12 & -5.87 & 0.1 \\
\hline $\mathrm{CE}-0$ & -4.51 & -8.75 & -4.32 & -2.74 & 1.49 & -0.24 & 0.61 & -0.14 & 0.15 & -7.46 & -4.01 & 1.3 \\
\hline CE-O & -4.85 & -9.44 & -3.53 & -3.41 & 3.5 & 0.78 & 0.15 & -0.48 & -0.31 & -7.54 & -4.64 & 1.32 \\
\hline CE-07-08-MB14 & -3.51 & -6.43 & -1.39 & -2.60 & & 1.95 & 0.28 & 0.09 & -0.18 & -6.19 & 0.21 & 4.4 \\
\hline 15 & -3 & -7.66 & -2.78 & -2.06 & & 2.24 & 0.68 & 0.74 & 0.23 & -6.01 & -0.70 & 36 \\
\hline CE-O & -4.19 & -8.06 & -2.05 & -3.33 & & 0.64 & & -0.38 & 0.02 & -7.43 & -4.08 & \\
\hline CE-O & -4.35 & -8.45 & -3.68 & -3.59 & & 1.01 & 0.25 & -0.72 & -0.20 & -7.32 & -3.69 & 2.1 \\
\hline CE-C & -3.31 & -6.21 & -3.68 & -2.42 & & 1.93 & 0.34 & 0.26 & -0.11 & -6.36 & -1.46 & 2.6 \\
\hline B19 & -1.81 & -3.49 & -1.22 & -1.46 & & -0.07 & 48 & 0.62 & 0.02 & -4.84 & -1.07 & 2.68 \\
\hline CE- & -2.94 & -6.07 & -2.05 & -2.60 & & -0.01 & 0.03 & 0.08 & -0.44 & -7.10 & -0.54 & 4.07 \\
\hline 21 & .05 & -6.09 & -2.79 & 2.88 & & 0 & & .33 & .43 & -7.00 & 43 & 4.75 \\
\hline $\mathrm{CE}-\mathrm{C}$ & -3.17 & -6.74 & -3.19 & -3.04 & & & & 0.90 & 0.34 & -7.45 & -0.57 & \\
\hline CE-O & -4.40 & -8.80 & -3.30 & -3.55 & & 0.66 & 0.19 & -0.56 & -0.27 & -7.49 & -3.99 & $1.8 \mathrm{~s}$ \\
\hline CE-C & -3.08 & -4.96 & -0.37 & -1.90 & & 1.11 & 0.63 & 0.16 & 0.19 & -3.81 & -0.50 & 3.4 \\
\hline CE- & & & & -2.29 & & -0.71 & & -0.02 & 44 & -4.84 & -2.71 & 20 \\
\hline & -3.80 & -6.68 & -1.80 & -1.80 & & & & & 19 & -4.46 & -1.72 & 2.67 \\
\hline 110 & -5.03 & -9.69 & -3.78 & -3.23 & 0 & -1.20 & 0.52 & 0.58 & 0.07 & -6.48 & -3.65 & $1.7 \mathrm{~s}$ \\
\hline CE-C & 0.08 & 0.59 & 1.64 & -2.15 & & 3.04 & -0.26 & 0.06 & -0.71 & -5.11 & -1.67 & $1.2 \varepsilon$ \\
\hline & -4.08 & -7.46 & -1.81 & -2.86 & & 0. & & & 0.06 & -5.97 & -2.10 & 2.56 \\
\hline & -3.17 & -5.64 & -2.87 & -2.16 & & 0.31 & & & & & -6.34 & -1.70 \\
\hline & .85 & -9.08 & -4.16 & -3.4 & & & & 49 & & -6.72 & -3.91 & 63 \\
\hline 12 & -4.15 & -8.11 & -4.25 & -2.63 & & 1.00 & & & 03 & -7.30 & -1.74 & 3.07 \\
\hline 313 & -4.80 & -9.17 & -4.07 & -2.96 & & -0.64 & & 0.09 & 0.01 & -7.16 & -4.00 & .54 \\
\hline & -4.50 & -8.35 & -4.13 & -2.33 & & -0.37 & & & 01 & -6.11 & -3.55 & 1.51 \\
\hline & & & & & & & & & & & 12 & 4.50 \\
\hline & - & -8.41 & -3.09 & -3 & & & & 23 & -0.05 & -7.47 & -4.16 & 35 \\
\hline & 1.79 & -9.31 & -3.3 & -3.28 & & -0.26 & & 0. & 66 & -7.26 & -5.05 & 80 \\
\hline & -1.94 & -3.87 & -2.62 & -1.62 & & & & & 02 & -5 & 0.27 & 9 \\
\hline & -1.95 & -3.71 & -1.27 & -1.6 & & -0.51 & & & 0.02 & -5.10 & -2.25 & .73 \\
\hline & - & -4.45 & - & -2 & & & & & & & $2 \mathrm{~s}$ & .08 \\
\hline & & -4.08 & & & & & & & & -5 & .87 & 3.07 \\
\hline & & -4.2 & & & & & & & & & 18 & .0 \\
\hline & -1.98 & -4.51 & -2.38 & -2.52 & & 1.36 & & 2 & -0.12 & -7.45 & 0.12 & 4.12 \\
\hline & -2.84 & -5.78 & 0.4 & -2.5 & & -0.34 & & & 16 & -7.29 & -0.95 & .59 \\
\hline & & & - & 1.8 & & & & & & -3 & & 0.41 \\
\hline & & 9 & & 7 & & & & & & & -5.80 & -0.11 \\
\hline & & & & & & & & & & -4 & & 0.92 \\
\hline & -4.3 & -8.1 & $-1 . c$ & -2.9 & & -1 & & & & -6.25 & -2.94 & 22 \\
\hline & & & & 2.08 & & & -0 . & 25 & 0.57 & -4.38 & -1.97 & 0.40 \\
\hline & & -0.3 & & $1 . \varepsilon$ & & & & & & -4.06 & -2.74 & 0.74 \\
\hline & & -9.16 & -3.18 & -3.0 & & .33 & & & & -5.87 & -4.87 & 0.66 \\
\hline & & -5.2 & -2.2 & -2.1 & & & & & & -5.00 & -6.28 & -1.67 \\
\hline & & & & & & & & & & & & 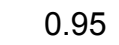 \\
\hline & 77 & -9.2 & & & & -3.2 & & & & -7.23 & -4.38 & 23 \\
\hline & 60 & -8.79 & -3 & -3. & & & & & -0.08 & -7.32 & -2.86 & .5 \\
\hline & 7 & -6.6 & - & -2 & & & & & 20 & -6. & -0.01 & 3.97 \\
\hline B15 & & -7.81 & -3 & -2.57 & & -0.7 & & & 0.22 & -6.95 & -3.39 & 1.67 \\
\hline & & & & & & & & & & & & .73 \\
\hline & & -8.95 & & -2.77 & & & & & & -7.25 & -3.63 & 1.75 \\
\hline & & -5.0 & & & & & & & & & -2.20 & .16 \\
\hline & -2.17 & -4.23 & -1. & -1.5 & & & & & 0.02 & -5.09 & -2.41 & .62 \\
\hline -169-MB20 & -2.69 & -5.62 & 1.86 & -2.67 & 0.53 & -0.72 & 0.11 & 0.04 & -0.35 & -7.48 & 1.11 & 0.4 \\
\hline
\end{tabular}




\begin{tabular}{|c|c|c|c|c|c|c|c|c|c|c|c|c|}
\hline & i_Cal & si_Dol & si_Sid & si_Gyp & i_Hem & i_Goe & si_Qtz & si_Bar & si_Cha & si_Hal & si_Illi & ii_Kao \\
\hline CE-08-170-MB21 & -4.33 & -8.46 & -3.35 & -3.27 & -1.85 & -1.91 & -0.19 & -0.58 & -0.65 & $\overline{-7.26}$ & -3.81 & \\
\hline CE-08-1 & -2.44 & -5.25 & -2.69 & -2.79 & & 0.81 & -0.01 & -0.87 & -0.47 & -7.51 & 0.49 & 4.67 \\
\hline CE-08- & -5.12 & -10.06 & -4.03 & -3.61 & -0.47 & -1.22 & 0.06 & -0.42 & -0 . & -7.58 & -8.42 & -1.41 \\
\hline CE-08-171-P1D & -3.15 & -5.03 & -0.45 & -1.87 & 3.00 & 0.51 & 0.59 & 0.18 & 14 & -3.83 & -3.87 & 0.50 \\
\hline CE-08-172-P1S & -5.95 & -10.55 & -3.62 & -2.25 & 0 & -0.87 & 0.85 & -0.02 & 40 & -4.25 & -6.61 & -0.67 \\
\hline$-P 2 D$ & -2.58 & -4.31 & -0.25 & -1.98 & 5.12 & 1.57 & 0.48 & 0.21 & 0.04 & -4.66 & -2.08 & 1.73 \\
\hline -D14 & -6.57 & & -4.18 & -3.67 & -1.71 & -1.85 & 0.67 & & 23 & -7.17 & & \\
\hline --JTC & -5.67 & -10.50 & -4.55 & -2.85 & -2.23 & -2.1 & -0.11 & 0.16 & -0.57 & -6.16 & -5.15 & 0.97 \\
\hline $\mathrm{BH} 10$ & -4.11 & -7.64 & -2.06 & -3.79 & 0.36 & -0.81 & 0.49 & -1.2 & .04 & -6.69 & -2.31 & 2.75 \\
\hline CE-09 & -4.24 & -7.66 & -2.27 & -2.50 & & 0.59 & & 0.44 & & -5.49 & -3.00 & 1.77 \\
\hline CW & -2.98 & -5.21 & -2.67 & -2.14 & & 0.73 & & -0.27 & 14 & -5.13 & -6.30 & -1.71 \\
\hline $2 \mathrm{D}$ & .51 & -4.18 & -0.18 & -1.98 & & 2.23 & & 0.06 & 05 & -4.66 & -1.05 & 2.55 \\
\hline AB11 & -4.35 & -8.24 & -4.62 & -3.24 & & -0.52 & 31 & -0.37 & 0.13 & -6.49 & -4.19 & 1.35 \\
\hline CE-OS & -4.65 & -9.00 & -4.75 & -2.40 & 1.4 & -0.27 & 0.68 & 0.09 & .25 & -7.05 & -2.87 & 2.18 \\
\hline IB-13 & .03 & -9.71 & -4.37 & -3.39 & & 0.67 & 0.32 & -0.58 & -0.11 & -7.57 & -3.44 & 2.02 \\
\hline -MB14 & 2.48 & -4.37 & -0.26 & -1.63 & & 1.44 & & 0.82 & 0.02 & -5.20 & 0.99 & 4.57 \\
\hline CE-O & .58 & -7.11 & -3.13 & -1.77 & & & & 61 & 20 & -6.25 & -5.45 & -0.39 \\
\hline CE-O & 1.62 & -8.95 & -2.3 & -3.29 & & & & 0.38 & 17 & -7.40 & & \\
\hline EE-0 & -4.67 & -9.21 & -3.44 & -2.79 & & 0.35 & 0.70 & 0.11 & 0.26 & -7.21 & & \\
\hline E-OS & -1.96 & -3.89 & 3.06 & -1.71 & & 0.79 & & 0 & -0.04 & -5.88 & -1.62 & 2.32 \\
\hline & & -4.79 & -1.86 & -1.51 & & & & c & & -4.81 & -1.55 & 2.36 \\
\hline & & -6.34 & 1.81 & -2.10 & & & & & & -6.97 & & \\
\hline & & -4.8 & & -1.19 & & & & & & -5.85 & & \\
\hline S3 & -3.96 & -7.94 & -2.17 & -3.16 & & & 0 & -0.56 & -0.13 & -7.46 & & \\
\hline CE-O & -3.21 & -5.15 & -0.49 & -1.85 & & 1.9 & & -0.04 & .06 & -3.88 & & \\
\hline P1S & & & & -2.23 & & 0.48 & & & .41 & -4.33 & -7.06 & -1.01 \\
\hline & & & & -2.73 & & & & & & -4.54 & -5.84 & -0.12 \\
\hline & -6.36 & .74 & -3.96 & -2 & & -0.29 & & & & -6.14 & -4.89 & 0.51 \\
\hline & -6.06 & & -3.54 & -3.15 & & -0.4 & & & & -6.66 & -5.49 & 0.02 \\
\hline $\mathrm{H} 30$ & & & & -3.08 & & 0.29 & & & 53 & -6.98 & -6.81 & -0.63 \\
\hline CE-09-99-CH31 & -2.93 & -5.33 & -0.38 & -2.01 & & 2.22 & & 0.3 & 24 & -5.31 & -0.45 & 3.30 \\
\hline 00-LHQ & 0.39 & 1.30 & 1.45 & -3.74 & 5.7 & 1.87 & 0.46 & -0.9 & 0.02 & -4.57 & 4.22 & 5.36 \\
\hline 01-MW13 & & & -2.17 & 3.71 & 0.87 & 0.76 & 0.10 & 0.31 & -5.27 & -2.33 & 2.27 & \\
\hline UCE-U9-TU3-IVIBSUT & -0.43 & -0.61 & -2.07 & -1. & & & $-0.5+4-5$ & & -0.45 & -5.10 & & \\
\hline & -3.96 & -7.49 & 1.7 & 3.27 & -0.0 & -1.05 & 0.49 & 0. & 0.07 & -7.23 & 0.69 & 4.16 \\
\hline CE-09-107-D18 & -5.40 & -10.11 & -2.23 & -3.86 & 1.14 & -0.43 & 0.62 & -0.12 & 0.17 & -7.52 & & \\
\hline
\end{tabular}

